\title{
L’architecture de paysage moderne du campus de l'Université de Montréal
}

\section{The modern landscape architecture of the campus of the University of Montreal \\ La arquitectura de paisaje moderno del campus de la Universidad de Montreal}

\section{Nicole Valois}

Volume 56, numéro 158, septembre 2012

Version originale soumise en mai 2012. Version révisée reçue en novembre 2012.

URI : https://id.erudit.org/iderudit/1014550ar

DOI : https://doi.org/10.7202/1014550ar

Aller au sommaire du numéro

Éditeur(s)

Département de géographie de l’Université Laval

ISSN

0007-9766 (imprimé)

1708-8968 (numérique)

Découvrir la revue

Citer cet article

Valois, N. (2012). L'architecture de paysage moderne du campus de l'Université de Montréal. Cahiers de géographie du Québec, 56(158), 343-372.

https://doi.org/10.7202/1014550ar
Résumé de l'article

Le campus de l'Université de Montréal est composé de bâtiments et d'aménagements paysagers hautement significatifs, dont la présence contribue à faire comprendre son évolution spatiale. Parmi eux, les espaces libres des années 1960 méritent une attention particulière pour plusieurs raisons historiques : ils font l'objet d'une réflexion pour la toute première fois dans l'histoire du campus ; l'Université de Montréal vit une phase d'expansion sans précédent ; les disciplines de l'aménagement et la pratique de planification émergent au Québec. Cet article présente de manière contextuelle les principes de planification qui ont mené à leur réalisation par La Haye et associés, firme d'urbanisme de premier plan au Québec. Il expose les qualités et les défauts des espaces libres de cette période moderne, soit l'innovation sur le plan de la vision planificatrice et du design, d'un côté, et la trop grande importance accordée aux automobiles au détriment de la sécurité et de la convivialité, de l'autre côté.
Tous droits réservés (c Cahiers de géographie du Québec, 2012
Ce document est protégé par la loi sur le droit d'auteur. L'utilisation des services d'Érudit (y compris la reproduction) est assujettie à sa politique d'utilisation que vous pouvez consulter en ligne. 


\section{L'architecture de paysage moderne du campus de l'Université de Montréal}

\author{
The modern landscape architecture of the \\ campus of the University of Montreal \\ La arquitectura de paisaje moderno del \\ campus de la Universidad de Montreal
}

\author{
Nicole VALOIS \\ Faculté de l'aménagement \\ Université de Montréal \\ Nicole.Valois@umontreal.ca
}

\section{Résumé}

Le campus de l'Université de Montréal est composé de bâtiments et d'aménagements paysagers hautement significatifs, dont la présence contribue à faire comprendre son évolution spatiale. Parmi eux, les espaces libres des années 1960 méritent une attention particulière pour plusieurs raisons historiques: ils font l'objet d'une réflexion pour la toute première fois dans l'histoire du campus ; l'Université de Montréal vit une phase d'expansion sans précédent; les disciplines de l'aménagement et la pratique de planification émergent au Québec. Cet article présente de manière contextuelle les principes de planification qui ont mené à leur réalisation par La Haye et associés, firme d'urbanisme de premier plan au Québec. Il expose les qualités et les défauts des espaces libres de cette période moderne, soit l'innovation sur le plan de la vision planificatrice et du design, d'un côté, et la trop grande importance accordée aux automobiles au détriment de la sécurité et de la convivialité, de l'autre côté.

\section{Mots-clés}

Campus de l'Université de Montréal, planification de campus, espaces publics modernes, architecture de paysage moderne, Jean-Claude La Haye.

\section{Abstract}

The campus of the University of Montreal is based on a highly significant association of buildings and landscape design that contributes to our understanding of the evolution of the use of space in the campus environment. For a number of historical reasons, the open spaces of the 1960s deserve particular attention. In the context of unprecedented expansion experienced by the university at the time and the simultaneous coming to age in Québec of the disciplines of landscaping and planning, open spaces on the campus became, for the first time in the history of the university, the focus of serious reflection. This article describes the context influencing the planning principles applied by La Haye and Associates, one of Québec's leading urban planning firms, in designing these open spaces for the campus. We trace the strengths and shortcomings of modern thinking about open spaces - on the one hand, the innovative approaches used in planning and design, and, on the other, the overemphasis placed on car related needs, at the expense of safety and opportunities for social interaction.

\section{Keywords}

University of Montreal campus, campus design and planning, modern public spaces, modern landscape architecture, Jean-Claude La Haye. 


\section{Resumen à venir}

El campus de la Universidad de Montreal está constituido de edificios y de instalaciones muy significativos puesto que muestran la evolución espacial del complejo. Por diferentes razones históricas, los espacios libres organizados en los años 1960 merecen una atención especial: por la primera vez en la historia del campus universitario son objeto de reflexión; por una parte, la Universidad de Montreal atraviesa una expansión sin precedente y, por otra, las disciplinas de organización espacial y la práctica de planificación emergen en Quebec. Este artículo presenta contextualmente los principios de planeamiento sostenidos por La Haye y asociados, una empresa de urbanismo de renombre en Quebec. Las cualidades y defectos de los espacios libres de ese periodo moderno son expuestos, por un lado, la innovación de la visión planificadora y de diseño y, por otro, la enorme importancia dada a los automóviles en detrimento de la seguridad y la convivencia humanas.

\section{Palabras claves}

Campus de la Universidad de Montreal, planeamiento del campus, espacios públicos modernos, arquitectura de paisaje moderno, Jean-Claude La Haye.

\section{Introduction}

Le paysage du campus de l'Université de Montréal, par son implantation sur le flanc nord du mont Royal, est unique. La tour de l'édifice principal, située sur son sommet et entourée de verdure, fait partie des images de marque de Montréal, bien ancrée dans l'esprit des Montréalais et des visiteurs. Sans doute en raison du prestige qu'elle évoque, l'établissement l'utilise régulièrement à des fins promotionnelles, affichant ainsi symboliquement son statut de grande université de recherche au Canada ${ }^{1}$. La représentation de l'Université par la valorisation du bâtiment principal et de sa présence sur le mont Royal met toutefois en marge d'autres composantes du campus. Les édifices et aménagements paysagers construits à différentes époques, du fait de leur qualité architecturale et de leur capacité à représenter l'évolution de l'architecture et de l'architecture de paysage au Québec, ont en effet une grande valeur (Cameron et al., 2008 et 2010). Or, parmi les réalisations qui ont jalonné l'histoire du campus, certaines d'entre elles méritent une attention particulière, notamment les espaces publics des années 1960.

On trouve quelques ouvrages sur l'architecture et l'histoire du campus, dont ceux de Bizier (1993) et Gournay (1990), mais jusqu'à présent, aucune étude ne porte uniquement sur la constitution des espaces extérieurs. Pourtant, ceux-ci ont été l'objet d'une attention toute particulière dans les années 1960 en tant que composantes majeures

1 La tour de l'édifice de Cormier affichait, selon certains auteurs, l'affranchissement de la communauté intellectuelle francophone face à l'hégémonie religieuse du Québec de l'époque et le désir d'occuper le versant nord de la montagne, en opposition à l'Université McGill, située sur le versant sud (Gournay, 1990). 
de l'expansion du campus. Les raisons de cette lacune sont nombreuses. D’abord, il existe peu de documentation sur l'aménagement des espaces extérieurs au Canada à l'exception des revues destinées aux professionnels. Cela relève peut-être du peu de connaissances que nous avons de la profession d'architecture de paysage, laquelle a contribué à leur réalisation. Ensuite, comme pour la plupart des grands ensembles institutionnels et résidentiels, il est difficile de scinder l'étude architecturale des espaces publics du campus tant ils sont spatialement réunis. Les plus anciens sont d'ailleurs l'œuvre d'un seul et même concepteur. Toutefois, les ouvrages de Cameron et al. (2008 et 2010) ont montré qu'ils peuvent être examinés comme des entités à part entière, en particulier ceux qui ont été construits à partir des années 1960. Une telle perspective concorde avec l'œuvre de La Haye et associés, une firme d'urbanisme de renom ayant contribué à former l'image actuelle du campus, pour qui les espaces libres étaient une constituante de l'aménagement, au même titre que les bâtiments, la voirie et les boisés ${ }^{2}$. Les espaces autour des édifices de cette période ont été réalisés en grande partie indépendamment des édifices et, par conséquent, la documentation à leur sujet se prête à une analyse.

Le présent article porte sur les espaces publics de la période moderne du campus. En présentant les principes de planification qui ont mené à leur réalisation ainsi que le contexte dans lequel le plan directeur s'est réalisé (consolidation des disciplines, expansion du campus et de son secteur, modifications du système d'éducation au Québec), l'article tente de démontrer

- que les espaces publics du campus s'inscrivent dans un courant émergent de planification de campus en Amérique et de l'urbanisme et architecture de paysage au Québec,

- que les espaces publics témoignent d'une esthétique de l'architecture de paysage des espaces publics de cette période,

- que les espaces publics et le plan directeur qui le supporte ont été un moyen d'inscrire le campus dans son territoire.

O y verra également les défauts et les qualités des réalisations, notamment l'importance accordée à l'automobile et aux systèmes de circulation ayant engendré des contradictions entre la vision et la réalisation.

Cet article ne dresse pas un portrait complet de cette partie de l'histoire du campus puisqu'il porte principalement sur les grandes lignes du plan directeur et sur les espaces libres, et non sur l'architecture par exemple. Il soutient que, parmi les traits caractéristiques du campus, les espaces libres issus du plan directeur de la période moderne ont une valeur historique et esthétique qui mérite attention ${ }^{3}$.

2 Le terme "espaces libres» désignera dans cet article tous les espaces extérieurs du campus de l’Université de Montréal: les places, parcs, boisés, sentiers, réseau piéton, espaces de repos, de rassemblement ainsi que la voirie. C'est d'ailleurs le terme utilisé dans les plans directeurs de 1964 et 1968 du campus de l’Université de Montréal et celui qui a été adopté par l'administration publique de la Ville de Montréal dès le milieu du XXe siècle jusqu'au moins 1992. Pour plus de détails à ce sujet, voir Landry (1992).

3 Le contenu de cet article fait suite aux travaux de Cameron, Déom et Valois colligés dans deux ouvrages sur les valeurs architecturales et paysagères du campus principal. Voir Cameron et al. (2008 et 2010). Il prend source également dans la présentation que l'auteure a faite dans le cadre des conférences associées à l'exposition «Modernisme menacé» de la Faculté de l'aménagement, en février 2011. 


\section{Documentation}

Pour écrire cet article, en plus des ouvrages sur l'histoire de l'Université de Montréal et des campus en Amérique du Nord, nous avons analysé les deux rapports de JeanClaude La Haye datés de 1964 et 1968 constituant le plan directeur et portant sur le projet d'extension du campus, principalement les parties portant sur les espaces libres ${ }^{4}$. Pour compléter la compréhension des deux rapports, nous avons aussi examiné le Fonds d'archives Jean-Claude La Haye du Service des archives et de gestion des documents de l'UQAM, plus précisément la partie du lot 63P1 portant sur les plans d'ensemble de l'Université de Montréal. Les parties de ce fonds portant sur les aménagements spécifiques ont été exclues.

\section{Campus modernes et transformation de l'éducation dans les années 1960}

Partout en Amérique comme en Occident, les années d’après-guerre ont été marquées par de grandes transformations sociales, économiques et culturelles qui ont eu des conséquences sur l'enseignement et la planification des universités. Richard Dober, diplômé de Harvard, fut l'un des premiers à établir des principes de planification, au même moment où l'Université de Montréal amorçait son expansion. Il explique, dans son ouvrage Campus planning (Dober, 1963), la corrélation entre l'augmentation démographique, la croissance économique, l'expansion des villes et la création d'institutions et d'infrastructures incarnant un idéal de liberté, de démocratie, de mieux vivre et de mieux habiter. La richesse d'un pays, dit-il, ne se mesurait plus au nombre d'habitants, au capital et aux richesses, mais désormais à la capacité de produire et de se démarquer par les avancées scientifiques. Ainsi, les avancées technologiques des pays concurrents ont poussé les pays occidentaux à vouloir rehausser le niveau de formation et à revoir le système d'éducation supérieure, ainsi que son accessibilité, jusque-là réservée à une certaine élite de la société.

Au début des années 1960, le Canada, tout comme les États-Unis, vit une situation semblable. La clientèle dans les collèges et les universités augmente rapidement, forçant les planificateurs et dirigeants d'établissements à repenser les programmes et les espaces voués à l'éducation (Turner, 1984). Les besoins se heurtent rapidement au manque d'espaces sur les campus, mais aussi à l'absence d'outils et de techniques de planification de campus à long terme. Aux États-Unis, par exemple, le tiers des institutions n'ont pas de planification de développement à long terme. Pourtant, l'ampleur des demandes l'exige pour assurer la cohérence à long terme entre une vision, des attributs physiques et des aspects financiers. L'ouvrage de Dober (1963) démontre la complexité des conditions selon lesquelles la planification doit s'effectuer et suggère de rassembler les fonctions sous forme de modules afin de permettre aux campus de s'adapter aux changements. L'aspect physique d'un campus n'est pas l'unique réalité dans une planification, dit-il. Celle-ci relève d’aspects aussi variés que la gestion des aspects financiers, le choix d'une vision et le rôle d'une institution vis-à-vis de la société. L'inventaire de l'existant et l'analyse des besoins immédiats et futurs en matière d'espaces et d'éducation sont essentiels au bon fonctionnement. Ce qui peut nous paraître évident aujourd'hui était loin de l'être à cette période puisque,

4 Le plan directeur comprend en fait deux rapports et plans. On verra plus loin les raisons de ces deux plans. 
traditionnellement, les esquisses préparatoires à la construction visaient un idéal architectural répondant aux besoins du moment. Il s'agissait désormais de penser à des structures souples et adaptables aux changements; un défi de taille en termes de design et de gestion, en raison de nouveaux outils de projection mis à la disposition des planificateurs.

Tout en connaissant le même sort démographique et idéologique que le reste de l'Amérique, le Québec vit au tournant des années 1960 des changements sociaux particuliers dont les impacts touchent les universités. Les domaines de l'éducation, de la culture et de la santé, jusque-là sous le pouvoir de l'Église, sont pris en charge par l'État. La restructuration entreprise par le gouvernement, qu'on a qualifiée de «socialisme d'État», a mené également à la création de plusieurs institutions financières et à l'un des plus ambitieux projets du Québec, la nationalisation de l'électricité. En contrôlant l'une de ses plus grandes ressources, le Québec se donnait les moyens de mettre en œuvre les programmes sociaux qui allaient moderniser la société et éduquer la population afin d'élever le niveau d'instruction que demandait la révolution scientifique et technologique occidentale.

Ainsi, le gouvernement revoit l'ensemble du système d'éducation de la province dans le but de donner accès à l'éducation à tous. Pour ce faire, et voyant le nombre d'élèves dans les écoles augmenter sans cesse, il met sur pied en 1963 la Commission royale d'enquête sur l'enseignement dans la province de Québec, dont les travaux ont donné ce qu'on a appelé le rapport Parent (Québec, 1965-1966). À la suite d'une longue consultation à la grandeur de la province, le gouvernement répond aux recommandations du rapport et rend l'éducation accessible à tous, crée de nouveaux établissements scolaires et fonde le ministère de l'Éducation. À l'Université de Montréal, une des conséquences de ce mouvement s'est traduite par le rapatriement des programmes autrefois dispensés par les communautés religieuses, notamment les cours classiques et les écoles spécialisées. Ce fut le cas, par exemple, de la formation des éducateurs en enseignement préscolaire et primaire ainsi que des soins infirmiers. Pour accueillir les nouveaux programmes, plusieurs bâtiments ont été construits et d'autres ont été rachetés aux communautés religieuses, notamment les pavillons Marie-Victorin et Marguerite-Bourgeois et ceux de la Faculté de musique et de la Faculté de l'aménagement.

\section{Évolution spatiale des espaces publics du campus de l'Université de Montréal}

Chacune des phases de développement du campus de l'Université de Montréal est tributaire de mouvements socioéconomiques plus larges qui en ont marqué l'aspect physique actuel. Bien que chacune comporte des aménagements extérieurs, celle des années 1960 se démarque des autres, en raison de la réflexion avancée sur les espaces publics qui l'a accompagnée et de l'étendue de l'expansion du campus.

Les premiers aménagements extérieurs d'importance voient le jour dès la première phase de développement, alors que le pavillon principal, de l'architecte Ernest Cormier, s’implante sur la montagne à partir de 1923 pour s'éloigner de la turbulence urbaine ${ }^{5}$.

5 Après une interruption, la construction du pavillon principal s'achèvera en 1943. Ensuite, l'édifice de la Polytechnique sera construit en 1958-1960, la Résidence «A», en 1956 et le centre culturel J.-A. De Sève, en1956, lesquels s'accompagneront d'aménagements extérieurs. 


\section{Figure 1}

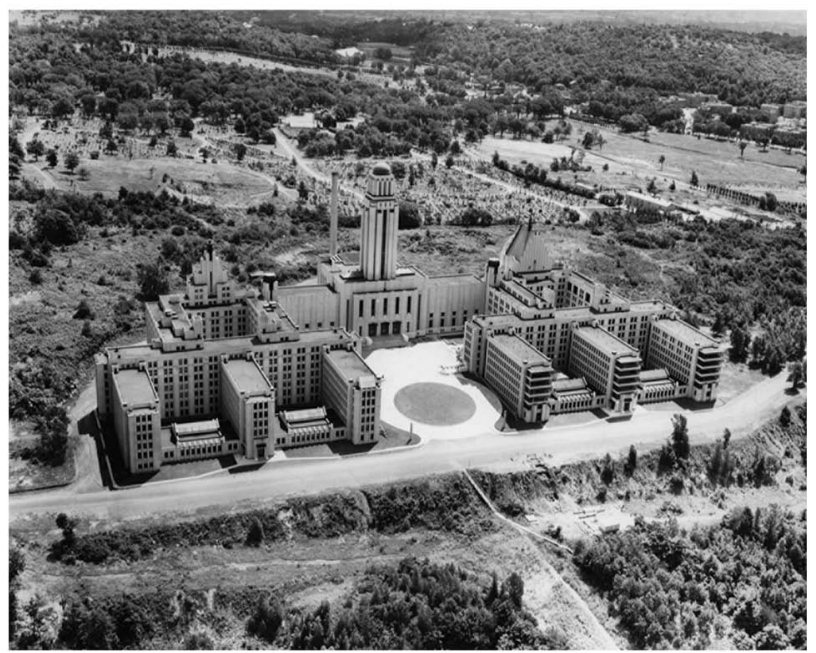

Sur cette vue aérienne du pavillon principal conçu par Ernest Cormier, on remarque la composition symétrique qu'accentue la Cour d’honneur.

Date de la photo: 25 juillet 1948

Source: Service de la gestion des documents et des archives, Université de Montréal, Fonds du Service des archives (D0036) 1FP1973. Photographe Surveys Limited.

\section{Figure 2}

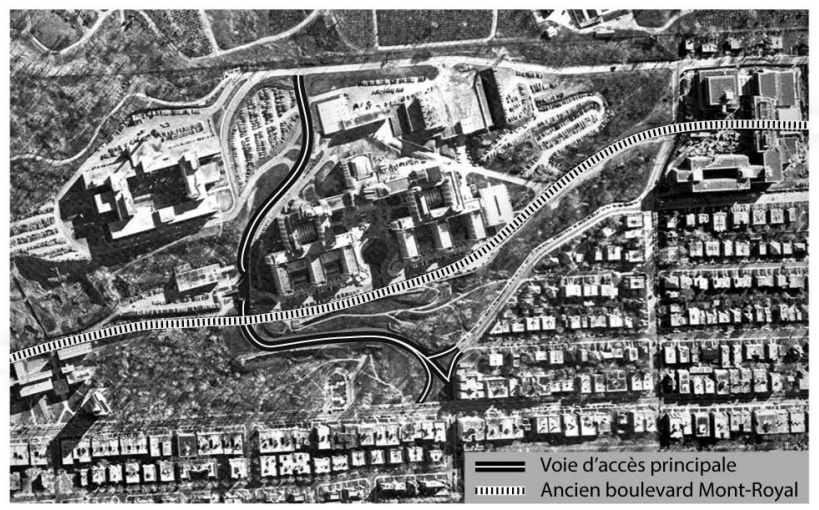

Le chemin de la Rampe remplace le boulevard Mont-Royal comme principal accès au pavillon principal.

Source: Université de Montréal - Plan d'ensemble [entre 1960 et 1980].

Université du Québec à Montréal. Service des archives et de gestion des documents. Fonds d'archives Jean-Claude-La-Haye, 63P1/734 (27.00)
La cour d'honneur du pavillon principal, défini par un cercle de verdure, l'axe de circulation automobile est-ouest, l'escalier de bois reliant l'actuel boulevard Édouard-Montpetit au pavillon principal ainsi que les boisés à l'état naturel font partie des premiers espaces libres. Hormis l'espace naturel, ils sont tous des aménagements "d'accompagnement» voués à des fonctions utilitaires ou de représentation (figure 1).

La composition se transforme radicalement entre 1960 et 1968 lors de la deuxième phase du développement où, pour répondre à l'augmentation de la population et à l'intégration des nouveaux programmes, de nombreux pavillons sont construits ${ }^{6}$. Les nouvelles voies de circulation asphaltées, les espaces entre les bâtiments, le réseau piéton intérieur et extérieur ainsi que les boisés constituent les espaces libres. La construction du chemin de la Rampe comme accès principal donne prépondérance à la circulation automobile intracampus et elle modifie radicalement l'image du campus (figure 2). L'importance des automobiles est à la fois le reflet de cette période où, partout en Amérique du Nord, s’accroît la dépendance à l'égard de son usage et une réponse à la volonté de l'Université d'intégrer le campus à la ville. Au système de circulation automobile se greffent les espaces libres entre les nombreux bâtiments dans le but de faciliter la

6 Ont été réalisés durant cette période le garage Louis-Colin, la Résidence «C», les pavillons SamuelBronfman et Liliane de Stewart. L'achat de bâtiments tels le pavillon de la Faculté de musique, la salle Claude-Champagne puis la Faculté de l'aménagement se concrétise également durant dette période. 
circulation piétonne (figure 3). Parmi les réalisations structurantes, se trouve la place de la Laurentienne dont la composition géométrique et l'emploi de formes hexagonales, de pavés à agrégats exposés, de madriers de bois et de pierres naturelles relèvent des tendances de cette époque.

\section{Figure 3}

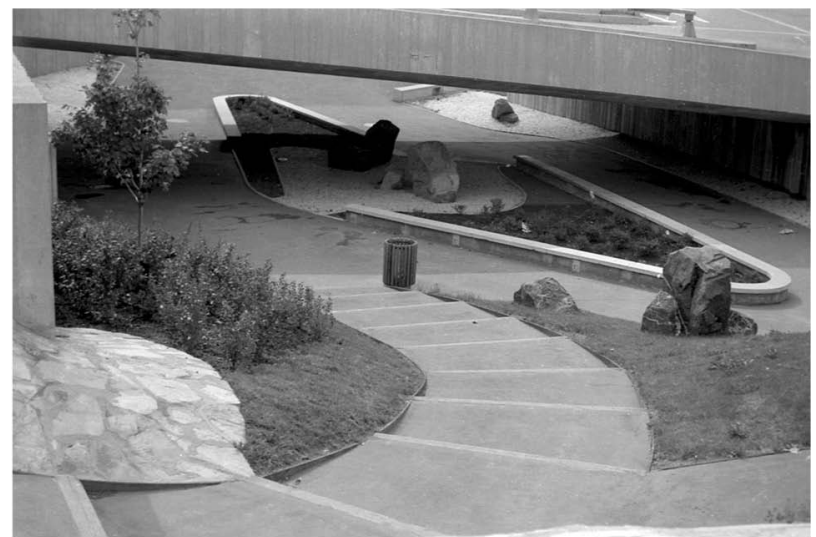

Ce passage entre le pavillon principal et l'École Polytechnique illustre le souci de paysager les liens entre les espaces du campus. Cet aménagement est en partie existant encore aujourd'hui.

Source: Université de Montréal - Plan d'ensemble [entre 1960 et 1980] Université du Québec à Montréal. Service des archives et de gestion des documents. Fonds d'archives Jean-Claude-La-Haye, 63P1/734 (27.00)

La période de 1995 à aujourd'hui, caractérisée par l'addition de plusieurs bâtiments liés aux sciences, a généré de nouveaux aménagements paysagers, réaffirmant les liens entre les espaces libres et répondant aux tendances stylistiques de l'époque ${ }^{7}$. C'est le cas de l'espace en paliers devant les pavillons Marcelle-Coutu et Jean-Coutu dont les formes, l'utilisation du béton et de l'acier ainsi que les végétaux s'inscrivent dans le mouvement contemporain de l'architecture de paysage. Les espaces publics réalisés par l'équipe de La Haye de la précédente période sont peu révisés durant cette période.

De cette évolution, les années 1960 et 1970 sont particulièrement importantes puisque c'est là qu'apparaissent les attributs physiques d'aujourd'hui et que s'est élaborée la réflexion sur les espaces libres (Cameron et al., 2010). Un des artisans importants de cette période est Jean-Claude La Haye qui, à titre de dirigeant de la firme Jean-Claude La Haye et associés, accompagnera l'Université dans la planification et la réalisation du campus. Son approche multidisciplinaire est mise au service de la planification alors qu'à cette même période, l'urbanisme et l'architecture de paysage sont en plein essor au Québec.

\section{Contexte de réalisation du campus des années 1960}

\section{Essor des disciplines de l'aménagement}

L'architecture de paysage et l'urbanisme sont des professions jeunes au Canada, dont l'essor coïncide avec l'expansion du campus de l'Université de Montréal. Jusqu'au milieu du siècle dernier, l'urbanisme et la planification étaient des pratiques marginales au Québec (Boisvert, 2003). Les programmes de formation étaient inexistants et ceux qui pratiquaient ces professions avaient été formés soit aux États-Unis, soit en Europe. Toutefois, tel que le mentionne Marsan (1974), il existait dès le XIX ${ }^{\mathrm{e}}$ siècle

7 Le plan directeur de Ouellet, Boisvert et Paré (1995) a guidé la dernière période d'expansion du campus. 
des opérations pour doter la ville d'un certain ordre spatial. Ainsi, selon des principes hygiénistes, un grand nombre de travaux ont vu le jour à Montréal pour pourvoir la population d'eau potable, d'infrastructures routières et de parcs récréatifs. Bien qu'au XX ${ }^{\mathrm{e}}$ siècle des développements sectoriels comme Ville Mont-Royal et la Cité du Tricentenaire aient vu le jour selon un plan, il ne s'agissait pas d'urbanisme à grande échelle comme aujourd'hui (Ibid.). Ce n'est qu'en 1944 que la Ville de Montréal s'est dotée d'un premier plan d'urbanisme, lequel n'a toutefois jamais été reconnu comme étant abouti (Ibid.).

C'est dire qu'avant le milieu du siècle dernier, Montréal et les grandes villes en général bénéficiaient de peu de moyens pour orienter l'évolution de leur territoire, et encore moins les universités. L'apprentissage de la planification des universités et des villes s'effectue pratiquement à la même période. Ainsi, plusieurs événements-clés de l'urbanisme au Québec coïncident avec le développement accéléré du campus de l'Université de Montréal. L'Institut d'urbanisme de l'Université de Montréal fut créé en 1961 ; l'Association des urbanistes du Québec fut fondée en 1963 et la Loi cadre d’aménagement a été créée en 1968, à laquelle d’ailleurs a participé La Haye.

Durant cette période également, l'architecture de paysage en tant que pratique reconnue prend son envol. Fondée aux États-Unis à la fin du XIX ${ }^{\mathrm{e}}$ siècle par Frederick Law Olmsted, créateur du parc du Mont-Royal à Montréal, la pratique apparaît au Canada au milieu du XIX ${ }^{\mathrm{e}}$ siècle alors que les horticulteurs et jardiniers anglais sont invités à concevoir des cimetières en Ontario et au Québec ${ }^{8}$. La pratique se diversifie par la suite vers la création de jardins publics et institutionnels, mais ce n'est qu'au début du XX siècle que Frederick Gage Todd, originaire d'Angleterre, et Charles Ernest Woolverton, d'Ontario, utilisent pour la première fois au Canada le titre d'architecte paysagiste (Fife et al., 2012) ${ }^{9}$. Progressivement, la profession s'organise à travers le pays: en 1934, la Société des architectes paysagistes et urbanistes est fondée, et le premier programme en architecture de paysage voit le jour en 1964 à Guelph, Ontario, suivi de près par Toronto, Winnipeg, Vancouver et Montréal. Elle connaît son véritable essor au milieu du $\mathrm{XX}^{\mathrm{e}}$ siècle, grâce aux grands projets comme Expo 67, le centenaire de la Confédération et la construction de la Voie maritime du Saint-Laurent (Paine, 1998). À Montréal, le programme d'architecture de paysage créé en 1968 acquiert son autonomie en 1978 en devenant l'École d'architecture de paysage de la Faculté de l'aménagement. L'Institut d'urbanisme de l’Université de Montréal étant déjà intégré à la Faculté, on peut penser que des liens se tissaient entre ces deux professions au moment de l'expansion du campus, dont la réalisation a fait appel à des experts de plusieurs disciplines.

\section{L'urbanisme à la Ville de Montréal}

L’idée de créer un système d'espaces libres à l’Université de Montréal a été précédée d'une vingtaine d'années par la Ville de Montréal pour son territoire, tel qu'en témoigne le premier plan directeur de Montréal. Ce plan montre de plus que l'urbanisme et l'architecture de paysage, bien qu'étant des pratiques embryonnaires, étaient interreliés dès la fin de la Seconde Guerre mondiale.

8 Frederic Law Olmsted fut le premier en Amérique à utiliser le titre d'architecte paysagiste en 1858, sur les plans de Central Park. L’année 1899 marque la fondation de l'American Society of Landscape Architecture ainsi que le début des cours en architecture de paysage à la Harvard School of Design.

9 On doit à Todd le plan de Ville Mont-Royal, l'aménagement du lac aux Castors dans le parc du MontRoyal et le Chemin de la croix de l'Oratoire Saint-Joseph. 
Après avoir créé en 1941 le Service d'urbanisme, la Ville de Montréal publie en 1944 le Plan directeur: urbanisation de Montréal. L'importance accordée aux espaces publics comme moyens de relier les lieux emblématiques de Montréal et d'offrir un meilleur cadre de vie constitue l'un des faits marquants de cet ouvrage. Le dessin d'un réseau vert continu, sur le plan d'accompagnement, montre les liaisons entre les espaces verts, les berges, la carrière Miron, le mont Royal et les «ceintures de verdure» propres aux futurs ensembles domiciliaires. La rue Rachel est montrée comme un lien vert important, unissant le parc Maisonneuve au mont Royal. Cette ceinture verte est expliquée comme étant le reflet de la vie sociale et l'importance de lier intimement les espaces libres, les édifices publics, les parcs et les écoles (Ville de Montréal, 1944).

L'importance accordée aux espaces verts est aussi manifeste dans le Plan directeur de Montréal: espaces libres, publié en 1955 par le Service d'urbanisme de la Ville de Montréal. Dans cet ouvrage, on analyse les besoins en regard de l'accroissement du territoire et de la population et il est proposé de répartir équitablement sur le territoire les terrains de jeux, de sport et autres. Durant cette période, plus précisément en 1953, la Ville de Montréal a engagé André Lafontaine, le premier architecte paysagiste (Cormier, 1990).

L'urbanisme et l'architecture de paysage dans les années d'après-guerre sont présentés par la Ville de Montréal comme deux domaines étroitement liés. Le plan de 1944 devance pour ainsi dire l'idée de la firme La Haye et associés de créer un système d'espaces libres reliés entre eux et cautionne en quelque sorte son approche de planification du campus visant à créer des lieux de rencontre et de verdure au profit du mieux-être des usagers.

\section{Jean-Claude La Haye}

Parmi le peu d'urbanistes de cette époque, Jean-Claude La Haye, qui fut le dirigeant de La Haye et associés et concepteur du campus, fait figure de leader. Par ses réalisations et son enseignement, il incarnait une vision de la pratique fondée sur l'atténuation des barrières entre l'urbanisme, l'architecture et l'architecture de paysage. Né en 1923 à Kapuskasing, dans le nord de l'Ontario, et formé en City planning à McGill, JeanClaude La Haye a amorcé sa carrière au sein du ministère des Affaires municipales, où il avait pour rôle de faire les premiers amendements à la Loi sur les cités et villes (Tellier-Cohen, 2003). Il est à l'origine de la création de l'Institut d'urbanisme de l'Université de Montréal, où il a enseigné trois ans, et de la création de la Corporation professionnelle des urbanistes du Québec, dont il a été le président fondateur jusqu'en 1968. Auteur de plusieurs plans directeurs d'urbanisme, il a eu une longue carrière jalonnée de nombreuses contributions au sein du gouvernement. Il a été directeur du comité d'aménagement, chargé de commenter le plan d'aménagement de la colline parlementaire de Québec, vice-président et président par intérim de la Commission de la capitale nationale et président de la Commission des biens culturels, de 1978 à 1980 . Une de ses plus importantes contributions fut son implication dans la Commission provinciale d'urbanisme, mieux connue sous le nom de Commission La Haye, pour laquelle il fut nommé président par le gouvernement en 1963 (La Haye, 1968b). La proposition de doter le gouvernement d'une politique d'urbanisme lui permettant de légiférer sur le développement donna lieu à plusieurs projets de loi, jusqu’à la création de la Loi sur l'aménagement et l'urbanisme, en 1979. 
Les réalisations professionnelles à l'actif des firmes qu'il a créées sont nombreuses. En plus du campus de l'Université de Montréal, on leur attribue le plan de mise en valeur de la région de Mirabel, ainsi que le schéma directeur de Sainte-Foy et son centre-ville. Le point de vue de Jean-Claude La Haye sur l'urbanisme peut se résumer par cette citation lors d'une entrevue: «Je pense qu'il faudrait arriver à satisfaire tous les besoins d'une vie d'homme à l'intérieur d'un quartier, microcosme de la société en général» (Tellier-Cohen, 2003 : 10). Il a fondé le premier bureau multidisciplinaire autour de 1965 alors que le premier rapport et les plans de l’Université de Montréal venaient d'être déposés.

\section{Expansion du campus dans les années 1960 et plan directeur}

la transformation du système de l'éducation, l'essor des disciplines de l'aménagement incluant l'urbanisme comme moyen de contrôle du développement au Québec, coïncident avec la croissance majeure qu'a connue l'Université de Montréal au tournant des années 1960. L'économie favorable et l'augmentation démographique sans précédent favorisent son expansion spatiale dans le but d'accueillir les nouveaux programmes et étudiants. Entre 1955 et 1965, l'effectif triple, les budgets de fonctionnement sont multipliés par six et ceux de la recherche, par dix (Archives UQAM-1). Des facultés sont créées et les départements se multiplient. Pour répondre à la demande en enseignement supérieur, les dirigeants éprouvent le besoin d'être guidés dans le développement spatial et souhaitent un plan flexible qui puisse s'adapter aux besoins futurs. L'Université de Montréal emboîte ainsi le pas à d’autres grandes universités américaines et canadiennes touchées par le même phénomène et commande un plan directeur.

Jean-Claude La Haye, semble-t-il mis au fait du projet, offre ses services dans une lettre adressée au recteur, Mgr Irené Lussier. Il y fait valoir son expérience et son approche multidisciplinaire en citant les membres de son équipe, composée des architectes Jean Ouellet et Victor Lambert, de l'architecte paysagiste Douglas Harper et de Raymond Archambault, arpenteur (Archives UQAM-2). Un projet d'expansion, dit-il, présuppose l'établissement d'un «plan d'ensemble». Il valorise son expertise en citant le projet du campus de l'Université de Sherbrooke, sur lequel il travaille alors. Quelques mois plus tard, le contrat lui est octroyé, et au début de 1961, le programme de travail est esquissé. Les plans sont déposés à l'Université de Montréal à la fin de 1963, et suivra en mars 1964 le dépôt du Rapport d'accompagnement du plan général d'aménagement de l'Université de Montréal (La Haye, 1964). Quelques mois plus tard, Gilles Gratton, ingénieur et directeur du Service des bâtiments et terrains de l'Université de Montréal, annonce les travaux. Parmi ces travaux, on compte la construction de l'édifice du service d'entretien des immeubles, d'un pavillon de salles de cours, de résidences d'étudiants, du stade d'hiver, des voies d'accès, de la rampe mobile ainsi que des raccordements aux services d'utilité publique. Ainsi, il a fallu moins de quatre ans pour planifier le développement du campus de la période moderne, l'une des plus marquantes de l'histoire de l’Université.

À la suite du dépôt de ces plans et du rapport, et après une première phase de construction, un deuxième plan sera réalisé en 1968. L'existence de ce deuxième plan, présenté dans un rapport intitulé Université de Montréal : le deuxième plan directeur (La Haye, 1968a), peut paraître étrange puisque le plan reprend en partie le contenu du premier et que la construction est déjà avancée en 1968. En fait, il découle de critiques formulées 
par la Ville sur le premier plan de 1964 à l'égard de son imprécision ${ }^{10}$. L’Université, dit-on, «s'engagerait formellement à [le] respecter» et, de plus, il prendrait «force de règlement, conformément aux nouvelles dispositions de la Charte de Montréal» (Archives UQAM-3 ${ }^{11}$. En réponse aux commentaires, ce deuxième plan directeur d'aménagement est rédigé, plus volumineux et plus détaillé. Le nombre de plans a triplé et l'analyse y est plus développée, notamment concernant la disponibilité de terrain et sa capacité d'absorption. La prise en compte du contexte urbain s'exprime davantage par la volonté d'avoir un campus à «l'échelle urbaine» avec une «unité esthétique», ainsi que par la conservation des qualités de la montagne.

Plus spécifiquement, les principes concernant les espaces libres, la circulation, le stationnement et l'unité esthétique architecturale sont approfondis selon une réévaluation des besoins et des relevés de terrain plus précis. Ainsi, l'idée d'une «colonne vertébrale» le long de laquelle se connectent les fonctions s'affirme en guise de vision d'un développement s'ajustant au gré des besoins. Les espaces libres y joueront un rôle important et le réseau piétonnier deviendra en ce sens une composante majeure, comme la circulation et le réseau souterrain dont, par ailleurs, le Comité consultatif d'architecture de l'Université de Montréal demanda formellement qu'il soit étudié ${ }^{12}$.

\section{La vision des espaces libres du plan directeur}

La vision spécifique aux espaces libres est très explicite dans le rapport de 1964, qui y consacre tout un chapitre, mais dont le contenu est repris dans celui de $1968^{13}$. Intitulé Organisation des espaces libres, ce chapitre définit les concepts de base et démontre en quoi les espaces libres incarnent les moyens d'atteindre les objectifs d'unification et d'intégration du campus à la ville.

On voulait que le nouveau campus se distingue de celui de Cormier; l'ampleur des besoins en espaces et en infrastructures allait en donner l'occasion. La vision générale se résume à une volonté de créer un campus plus urbain qui mette en valeur les attributs naturels du site au moyen d'un système interne dont font partie les espaces libres. Ce système permettrait de répartir les fonctions de programmes et les équipements autour d'un réseau piétonnier et automobile doté d'espaces publics et raccordé à la ville. La connexion à la ville s'incarnait au sens propre et figuré, c'est-à-dire en liant le chemin de la Rampe et le chemin de la Polytechnique aux rues de la ville et en ouvrant l'accès au campus aux communautés riveraines.

10 En effet, le rapport du Service d’urbanisme daté de février 1967 qualifie le plan d’imprécis, "sans programme» et sur lequel il est difficile de se prononcer (Archives UQAM-3). Il fait le point sur les propositions initiales de la Ville et sur certains manquements au plan directeur de La Haye, notamment le manque de liens entre le campus et le quartier. Ce dernier aspect constitue d'ailleurs « une nouvelle conclusion» dans le rapport de 1964 où dans le rapport de 1968 l'on propose que «l'expansion du campus» soit intégrée à la ville.

11 La modification de l'article 612 de la Charte de Montréal en 1965 stipulait que, pour l'obtention d'un permis de construire des grands ensembles, les propriétaires devaient soumettre un "plan d'ensemble» à l'approbation du Service d'urbanisme. Ce serait, selon plusieurs sources, l'ancêtre du Programme particulier d'urbanisme que l'on connaît aujourd'hui.

12 En effet, il demanda que soit étudiée «la possibilité d’un réseau de circulation couverte ou intérieure entre les immeubles et les groupes d'immeubles» (Archives UQAM-3).

13 Le contenu du chapitre sur les espaces libres du rapport de 1964 est dispersé dans le rapport de 1968 entre différentes sections. À noter que l'expression «espaces libres» fait référence à la «place d'étudiants et réseau piétonnier» sur le plan. 


\section{Un système unifié d'espaces}

La Haye et son équipe croyaient à la vertu sociale et fonctionnelle des espaces libres. Une grande importance était accordée au réseau que ceux-ci constituaient et à leur intégration au réseau automobile et piétonnier interne, dans un système unifié. D’un autre côté, ces espaces libres sont présentés comme des ponctuations dans le système, tels des espaces d'arrêt et de transition entre les édifices et des lieux de rassemblement servant à distribuer et lier les fonctions.

Les espaces libres ne sont pas des éléments résiduels du développement; ils ont une valeur positive et ont des fonctions à remplir : prolongement du caractère naturel de la montagne, transition avec l'entourage urbain, aires de promenade et de détente, aménagements sportifs, places de réunion. (La Haye, 1968a: 17)

Créer un réseau au cœur du campus afin de faciliter les rencontres et les échanges, «[...] distribuer les fonctions autour d'un pôle d'attraction qui forcerait les rencontres et les échanges d'idées", telle est l'aspiration (Ibid. : 5).

\section{Les fonctions des espaces libres}

L’importance accordée à la notion de fonction est notable, dans le rapport, par le vocabulaire utilisé pour qualifier les espaces libres. Les termes «transition», «lieux de rencontre», "aires de regroupements » désignent des lieux très précis. Ainsi, les aires de transition comprennent le parc autour de l'actuel édicule de métro; les aires de rencontre et de repos sont en périphérie des rues tandis que les aires de regroupement et de communication comprennent les espaces en bordure des édifices et des voies de circulation. Certains espaces regroupent plusieurs fonctions:

L'espace externe du bâtiment remplit une fonction complémentaire à celle de l'espace interne. Il prend une valeur positive par ses fins de circulation, d'accueil, de groupement, de détente ou d'intégration à la nature. (La Haye, 1968a: 42)

Figure 4

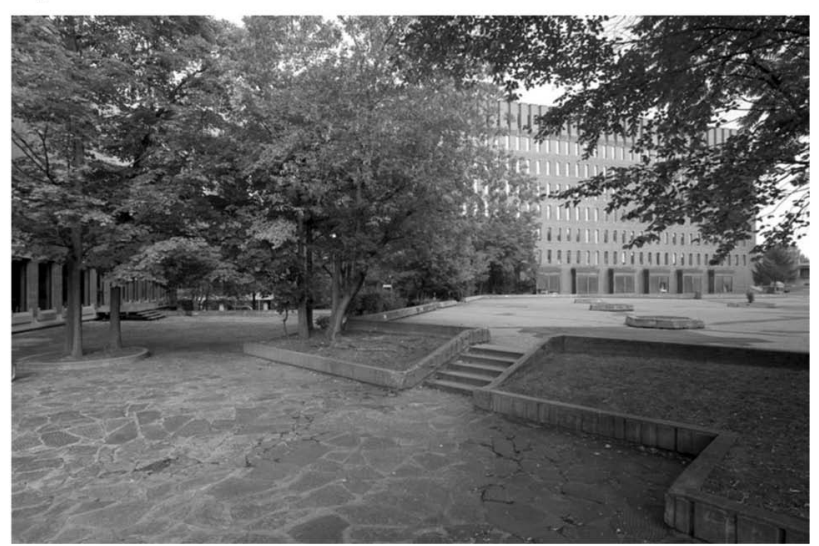

Section ouest de la place de la Laurentienne, aménagée en terrasse à proximité de la cafétéria. Le revêtement en pierres plates, les bordures et les escaliers sont d'origine.

Photo: Chaire de recherche du Canada en patrimoine bâti de l'Université de Montréal (CRCPBUM)
La place de la Laurentienne, le toit du garage Louis-Colin, la Cour d'honneur de l'édifice principal de Cormier de même que la devanture de ses ailes incarnent ce principe de multifonctionnalité. Ce sont des lieux de rencontre, de circulation et d'accueil, clairement identifiés comme tels au départ et qui remplissent bien leur rôle encore aujourd'hui (figure 4). Le positionnement varié des bancs en béton devant les ailes du pavillon Roger-Gaudry, par exemple, favorise le

rassemblement en petits groupes, dans un endroit fort emprunté par les usagers qui proviennent de la rampe mobile, à proximité (figures 5 et 6). 


\section{Figure 5}

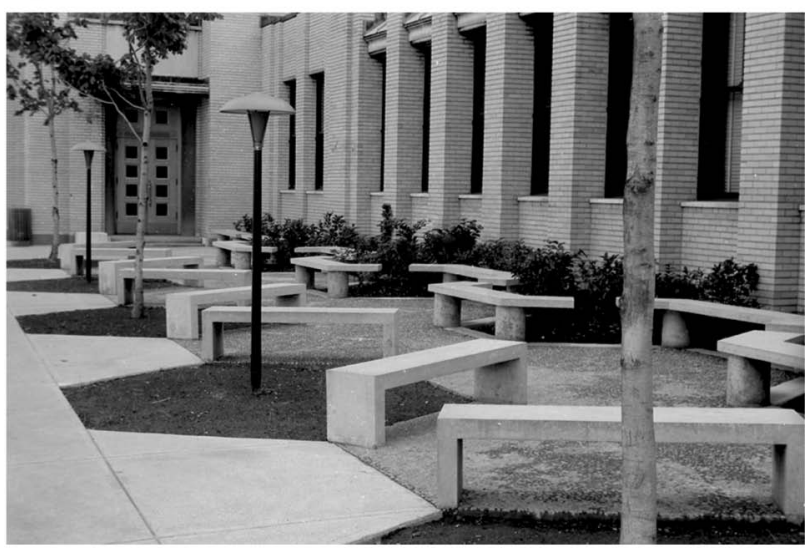

Conçu comme un lieu de rassemblement entre deux ailes du pavillon principal, cet espace est représentatif du design de la période moderne par l'utilisation de formes simples du béton, et du motif orthogonal.

Source: Université de Montréal - Plan d'ensemble [entre 1960 et 1980]. Université du Québec à Montréal. Service des archives et de gestion des documents. Fonds d'archives Jean-Claude-La-Haye, 63P1/734 (27.00)

\section{Figure 6}

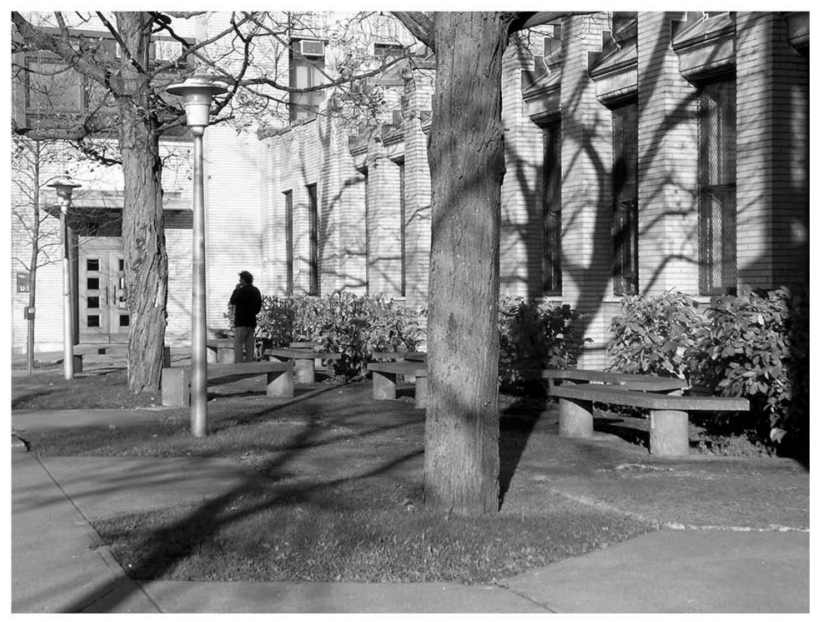

Par rapport à la photo d'époque précédente, on remarque que certains bancs ont été retirés, mais que l'espace garde le même esprit.

Photo: Nicole Valois, novembre 2011

\section{Les attributs naturels}

Les richesses naturelles du campus sont nombreuses et leur intégration au design des espaces libres permettrait de les mettre en valeur. Le «profil» créé par les nouveaux bâtiments devait s'adapter à la montagne, conformément au règlement de la Ville. Ainsi, la hauteur des bâtiments est soigneusement étudiée dans le rapport et l'intérêt des «composantes naturelles du campus» est signalé, ainsi que la nécessité de préserver les aires boisées. 
L'objectif $[\ldots]$ serait la réalisation d'un profil général comportant une intégration harmonieuse de bâtiments et d'espaces verts ou boisés qui ne défigure pas la montagne. (La Haye, 1968a: 28);

[...] les principaux objectifs du contrôle de développement [...] résident principalement dans la préservation du profil et du caractère naturel de la montagne, principal élément pittoresque de la métropole. (Ibid.)

La préservation des sous-bois par l'aménagement de sentiers faisait partie du plan dans la mesure où «sa mise en valeur et aussi sa régénération sont des considérations qui commandent une attention particulière dans la réalisation du campus» (La Haye, 1964: 30). On notera d'ailleurs que de nombreux sentiers traversant les boisés sont encore en fonction, notamment ceux qui sont situés en contrebas de l'édifice principal. L'inventaire général des boisés sur la planche intitulée Les espaces verts naturels et aménagés montrant les essences dominantes (chêne-érable, érable, frêne et robinier), leur état général et leurs calibres attestent de l'intérêt des éléments naturels.

\section{Le rôle des végétaux dans les aménagements}

Les végétaux ont le rôle d'accentuer le caractère naturel de la montagne, d'assurer les transitions au moyen d'un traitement harmonieux et d'assouplir les abords de route après la construction. Ainsi, le chemin de la Rampe suit la topographie de la montagne et aux abords, les arbustes ornent les talus. Les murs de soutènement le long de ce chemin et derrière le pavillon principal, conçus par l'artiste Jean-Noël Poliquin, com-

\section{Figure 7}

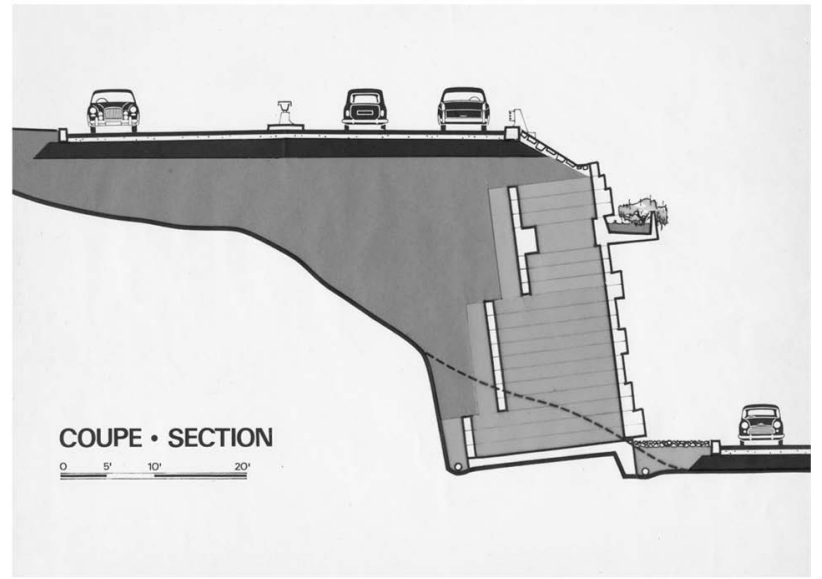

Détail en coupe du mur de soutènement, montrant l'inventivité de l'ouvrage sur le plan structural et architectural.

Source: Université de Montréal - Plan d'ensemble [entre 1960 et 1980]. Université du Québec à Montréal. Service des archives et de gestion des documents. Fonds d'archives Jean-Claude-La-Haye, 63P1/734 (27.00) prennent des caissons en béton préfabriqué pouvant contenir des plantes: tout en répondant à des besoins structuraux, l'assemblage des modules procure un jeu d'ombres et de lumière qui assouplit l'effet de dénivellation important devant le pavillon principal (figures 7 et 8). L'impact des murs à ériger pour des besoins de construction est allégé par la plantation. Aujourd'hui, les murs sont encore très présents, mais les plantes n'y sont plus et l'escalier est condamné tandis qu'ailleurs plusieurs végétaux sont toujours présents. 
Figure 8

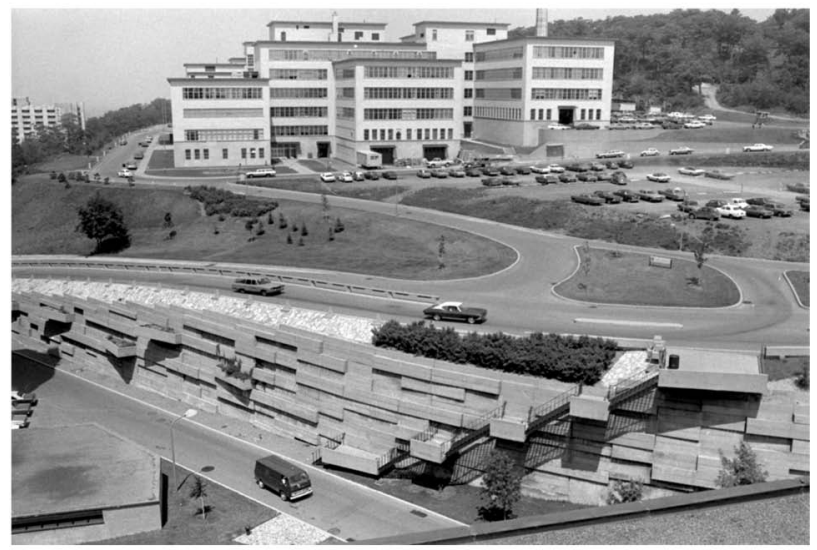

La photo montre le mur de soutènement dessiné par l'artiste Jean-Noël Poliquin et son inscription dans la pente de la montagne et autour des voies automobiles.

Source: Université de Montréal - Plan d'ensemble [entre 1960 et 1980]. Université du Québec à Montréal. Service des archives et de gestion des documents. Fonds d'archives Jean-Claude-La-Haye, 63P1/734 (27.00)

\section{L'intégration du campus dans la ville}

À travers son histoire, le rapport qu'entretient le campus avec l'urbain est lié au développement du secteur, mais il est difficile à ce stade de nos recherches de départager lequel a structuré l'autre. L'on peut affirmer, toutefois, que l'Université souhaitait au départ un campus calme, loin de l'animation urbaine, et que les aménagements des années 1960 avaient pour but de répondre au souhait de la Ville d'en faire un campus plus intégré à la ville. Le choix du site, comme nous l'avons vu, découle de la volonté de l'administration d'éloigner le campus de l'activité de la ville. Le flanc nord de la montagne, encore inexploité, et le secteur de Côte-des-Neiges n'ayant pas été rejoints par le développement de Montréal sont alors des facteurs de choix pour conférer au campus un caractère rural. À la suite de la première phase de développement, au milieu des années 1950, le secteur se transforme. Le campus participe à ces changements par son expansion et devient un campus à caractère urbain même si, dans les faits, les accès et les circulations sont sur la propriété de l'Université. Tout le secteur s'urbanise, y compris l'Université. La disparition presque définitive du noyau villageois au début des années 1960, au profit de la construction de tours résidentielles et de la reconfiguration de l'intersection des chemins Côte-des-Neiges et Queen-Mary, ainsi que l'achat des rues avoisinantes par l'Université pour construire les pavillons des sciences humaines dans la partie ouest ont contribué à modifier le tissu urbain aux pourtours de l'Université (Garcia et Garcia, 2006). La Haye reconnaît que, par ses intentions, le campus de Cormier se transforme au rythme de la densification du secteur : «[il est] aujourd'hui au centre géographique du développement métropolitain» (La Haye, 1968a: 24). L’urbaniste ira jusqu'à suggérer des changements de zonage pour le boulevard Édouard-Montpetit afin de permettre l'installation de commerces au profit de la communauté universitaire, chose qui ne se réalisera pas ${ }^{14}$. La centralité du campus, peut-on en déduire, est certes le fait de sa position géographique dans la ville. Mais elle prend sens également en regard du développement autour tout autant que de l'expansion de l'Université. D'une certaine manière, cette citation sur la centralité du campus présumait que ce campus faisait dorénavant partie d'une dynamique urbaine sociale et spatiale. La variété et l'accessibilité par le public des espaces aménagés en bordure du boulevard ÉdouardMontpetit, les chemins d'accès connectés au réseau de la ville et la mise en valeur du caractère naturel de la montagne servent d'arguments pour appuyer que l'intégration

14 Jean-Claude Marsan réitère cette idée en proposant de densifier le campus, dans plusieurs articles, notamment celui publié dans la revue Forum (Marsan, 2009). 
du campus à la ville est bel et bien réelle. Une attention toute particulière a en effet été accordée à l'implantation des édifices et espaces verts de manière à ce qu'ils participent positivement à l'ambiance du quartier et qu'ils s'inscrivent dans le profil de la montagne. L'emplacement des fonctions culturelles et sportives en bordure du boulevard Édouard-Montpetit appuie cette volonté puisque cela attirera la population environnante. L'intégration, toutefois, ne serait pas pleinement réussie pour certains, dans le sens où l'Université n'a pas saisi les occasions de développement qui se présentaient lors de son expansion. Elle restera pour eux un campus refermé sur lui-même ${ }^{15}$.

\section{Figure 9}

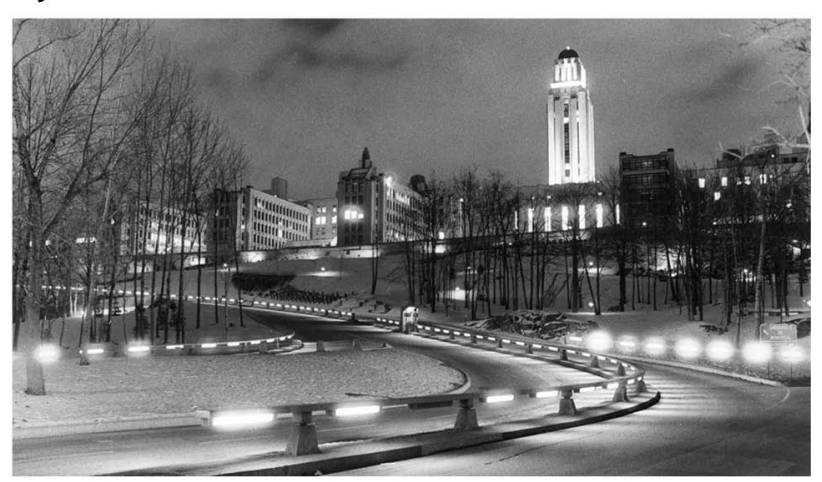

La rampe d'accès au pavillon principal a été l'un des tout premiers ouvrages de la deuxième période de développement du campus. Conçue à l'image d'un parkway, elle a mérité le prix d'excellence du « Highway Beautiful » de la «Canadian Good Road Association » en 1970.

Source: Université de Montréal - Plan d'ensemble [entre 1960 et 1980].

Université du Québec à Montréal. Service des archives et de gestion des documents. Fonds d'archives Jean-Claude-La-Haye, 63P1/734 (27.00)

À défaut, pour certains, d'avoir un campus pleinement intégré à la ville, le caractère urbain moderne est fortement incarné par le design des espaces libres, y compris par les voies de circulation interne. Le chemin de la Rampe, réalisé en 1965, évoque cette image et constitue une composante-clé du design de cette période. Son tracé sinueux gravit la pente depuis le boulevard Édouard-Montpetit jusqu'au pavillon principal, en ouvrant des vues vers la ville, tels les parkways américains, dont le tracé suit des règles de mise en valeur des vues sur le paysage (figure 9). Ce système, bien qu'il soit connecté au réseau urbain, est resté accessible aux usagers du campus jusqu'à ce qu'on abandonne les guérites au profit des barrières automatiques. Par ce geste, le chemin est-ouest, ouvert au public, est partiellement restitué par le lien entre la rue Decelles et le boulevard Édouard-Montpetit.

\section{Vision idéale et spatialité}

Les rapports du plan directeur du campus exposent la vision des espaces libres en véhiculant l'idée d'un campus convivial profitant des attributs naturels puis d'un campus urbain fonctionnant comme un système. Les plans qui accompagnent le plan directeur, par le dessin d'un système interne composé des réseaux piétonniers et automobiles et des espaces de rassemblement, concrétisent cette vision qui a guidé les aménagements actuels. Si l'on en juge par les plans concernant les espaces publics, on note toutefois un écart entre la vision écrite et la transposition spatiale en plan et en réel (figures 10, 11, 12).

15 Voir à ce sujet l'article de Jacques Trudel (2011) dans lequel il souligne l'autonomie dont jouit l'Université en termes de développement, laquelle nuirait à une pleine intégration du campus dans la ville. À noter que l'auteur a participé aux discussions entre la Ville et l'Université au moment de son expansion dans les années 1960, en tant qu'urbaniste au Service d'urbanisme. 

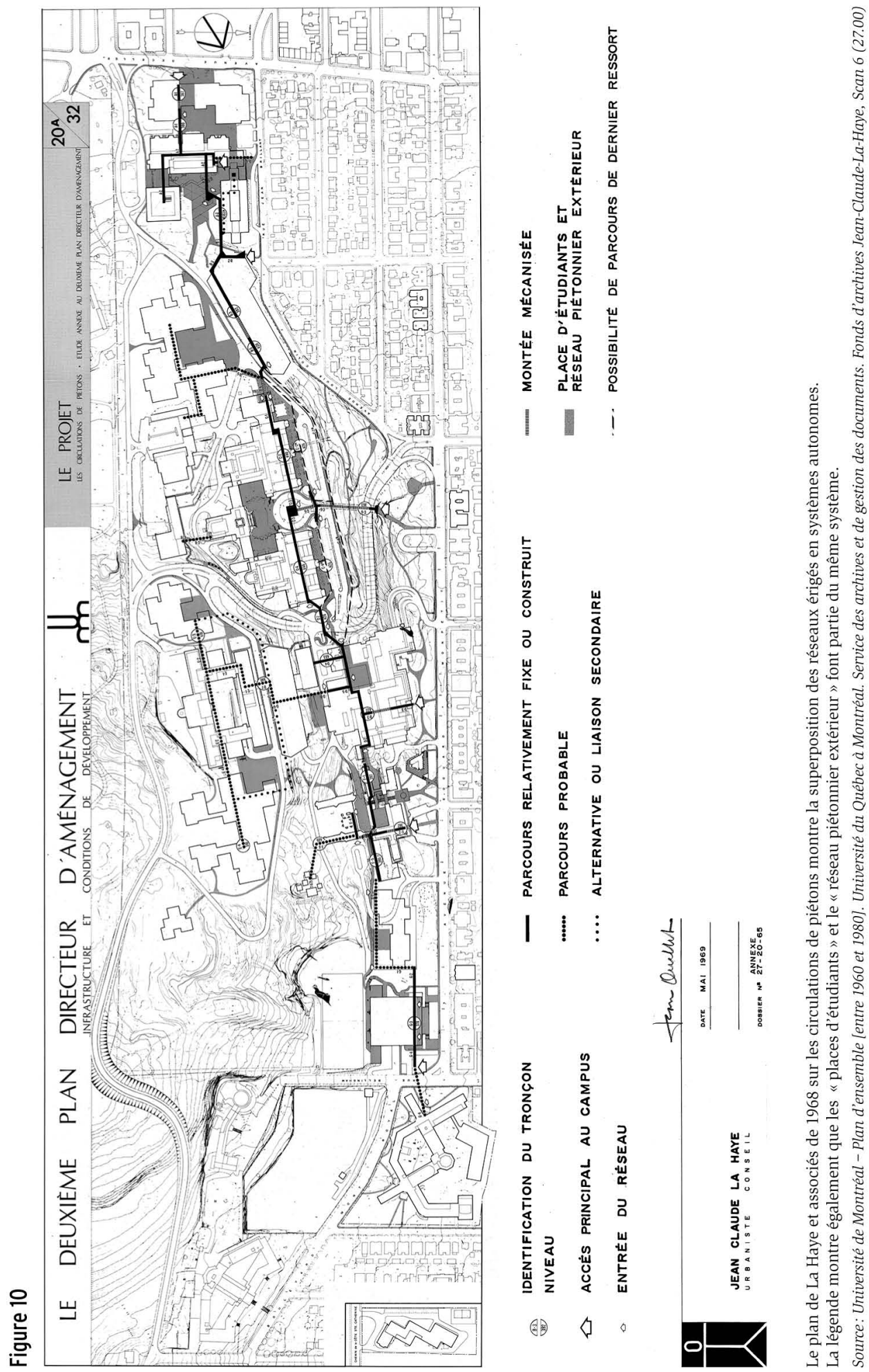

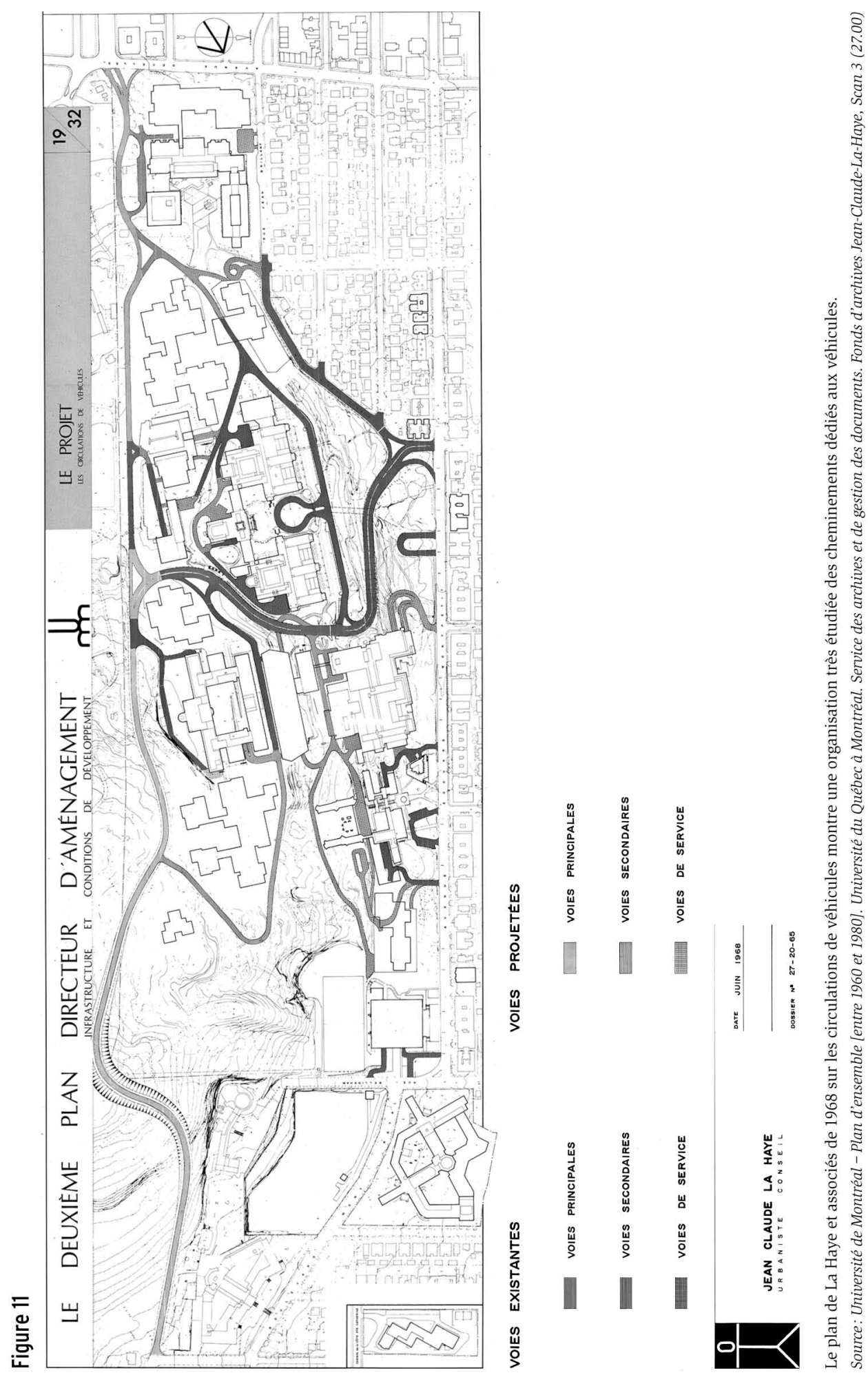

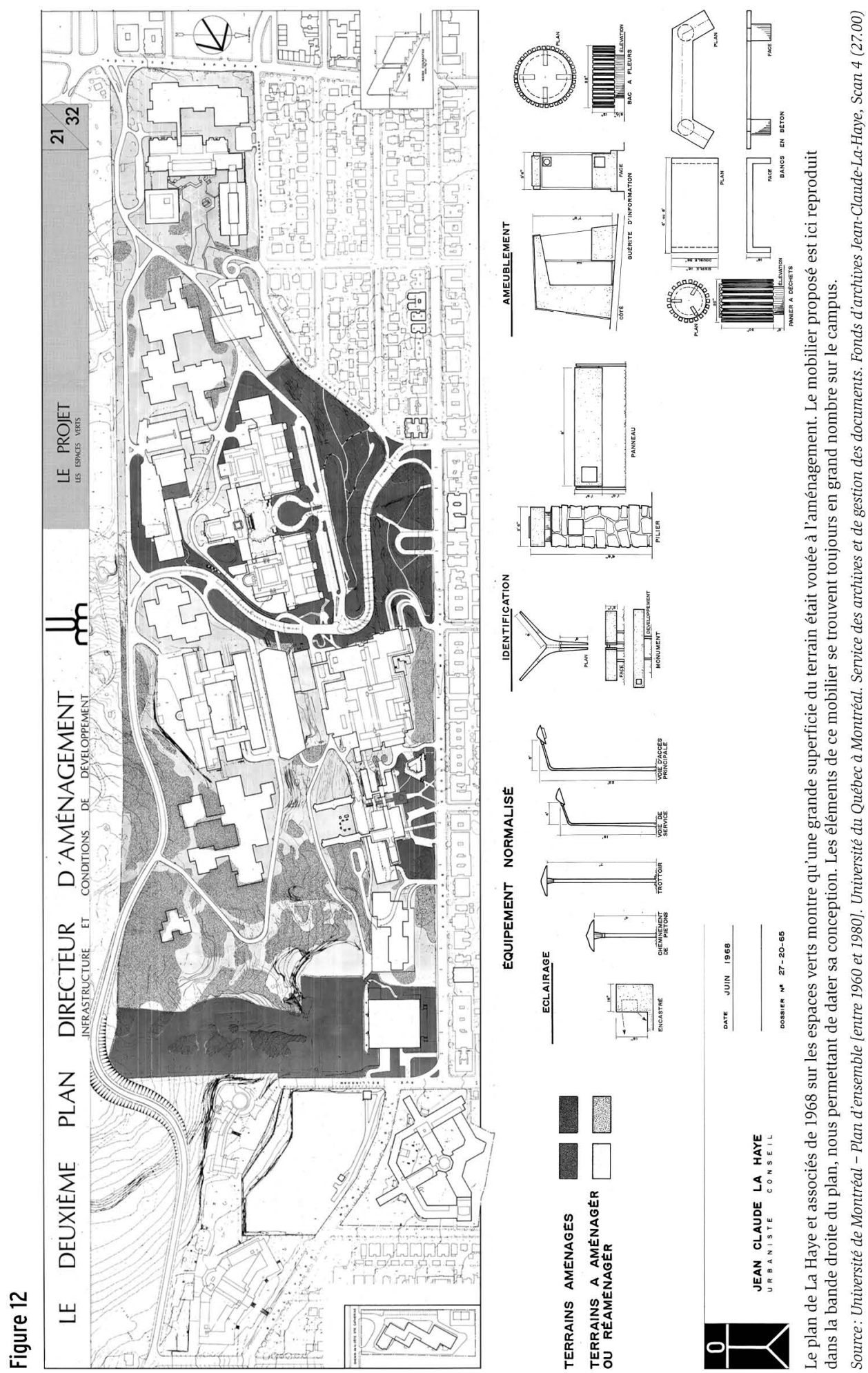
Le plan intitulé Le projet - les circulations de piétons illustre très bien le concept d'espaces libres, mais pourtant l'accent est mis sur un système ${ }^{16}$. L'accès au campus montré se fait sans hiérarchie par le réseau souterrain plutôt que depuis la rue. En effet, selon l'indication des flèches, on accède au campus depuis trois pavillons (HEC, Jean-Brillant et J.-A. De Sève), deux garages (Louis-Colin et le Stade d'hiver) et par la rampe mécanique. De plus, le système de "place d'étudiants et réseau piétonnier extérieur ", représenté en gris pâle sur le plan, comprend autant les résidus d'espaces que les véritables espaces publics. Le plan comporte peu d'indications sur la manière dont sont traitées les traverses de rue alors que certains sentiers et places se prolongent jusqu'aux trottoirs adjacents et que les accès piétonniers depuis la rue ne sont pas reconnus comme des entrées au campus.

Par ailleurs, la planche portant le titre de Le projet - les circulations de véhicules montre une voie de traverse est-ouest située à la limite sud du campus se prolongeant dans le boisé Saint-Jean-Baptiste (figure 11). Cette voie s'avérait une solution d'importance pour mieux intégrer le campus à la ville par le remplacement du chemin qui traversait le campus en passant devant le pavillon principal pour rejoindre le boulevard MontRoyal. En raison de sa faible faisabilité et en accord avec la Ville, seulement la partie ouest a été réalisée (chemin de Polytechnique) mettant un terme à une vision plus élargie d'intégration du campus à la Ville.

On notera que les bornes signalétiques en pierres de taille marquent principalement les entrées automobiles du campus, puis celle qui annonce l'entrée piétonne depuis l'avenue Jean-Brillant est excentrée par rapport au flux normal de la circulation (figures 13 et 14). Pourtant, les bornes et les guérites de l'«équipement normalisé» de la planche intitulée Le projet - les espaces verts (figure 12) semblent destinées à jouer un rôle important de marquage des entrées.

\section{Figure 13}

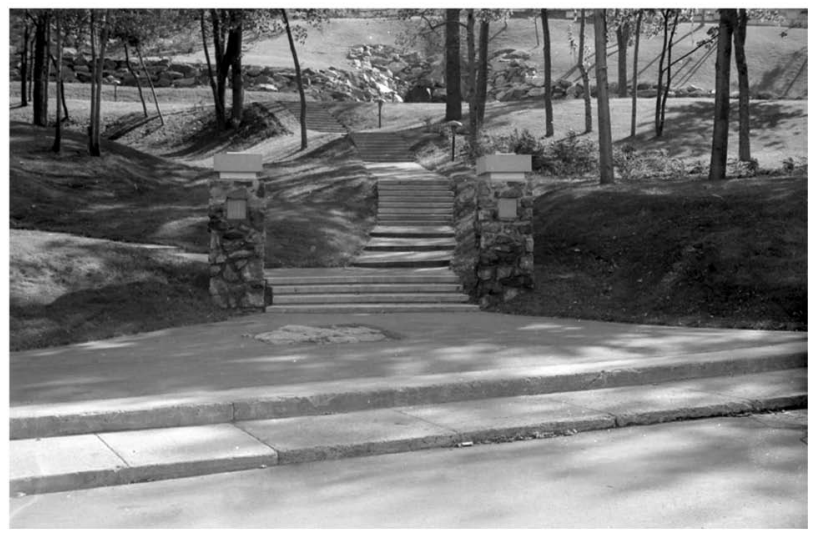

Les bornes de signalisation le long de l'avenue Louis-Colin indiquent l'accès piéton vers le haut du campus.

Source: Université de Montréal - Plan d’ensemble [entre 1960 et 1980].

Université du Québec à Montréal. Service des archives et de gestion des documents. Fonds d'archives Jean-Claude-La-Haye, 63P1/734 (27.00)
L'intention de séparer les fonctions piétonnes et véhiculaires, extérieures et intérieures, tout en créant un système unifié, peut avoir rendu la tâche ardue. Les plans dont nous venons de parler montrent la volonté de moderniser le campus à l'image des grandes villes et de répondre aux objectifs du Comité consultatif de l'Université d'«établissement d'une colonne vertébrale [...] sur lequel viendraient se greffer les différentes composantes du campus [...]» (Archives UQAM-4).

16 Il s'agit de la planche 20A/32. Une précédente, intitulée 20/32, avait été produite, mais sur cette dernière, les accès, la montée mécanique et la topographie n'apparaissaient pas. 
[...] à l'instar des villes anciennes dans lesquelles les places et les rues appartiennent aux foules et aux piétons, on voit se dessiner [...] des réseaux distincts rendant les diverses fonctions urbaines facilement accessibles aux piétons, leurs véritables usagers. (La Haye, 1968a: 44)

\section{Figure 14}

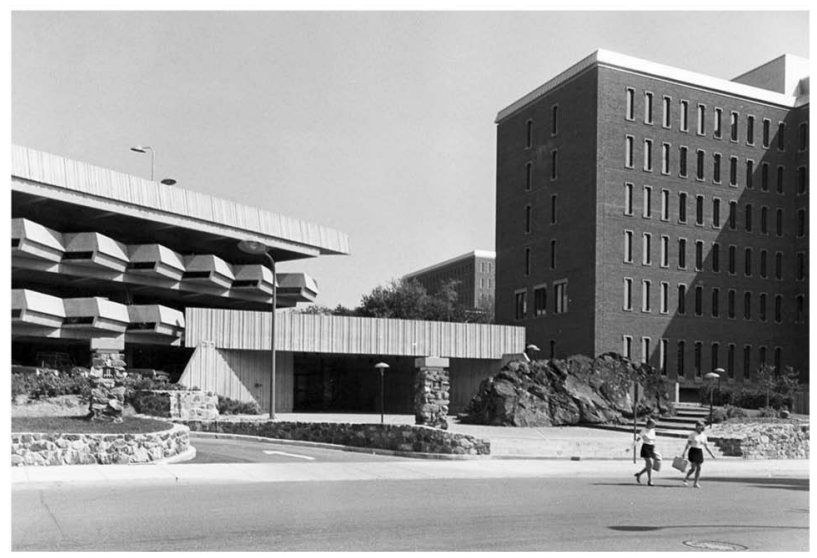

Les bornes situées à l'entrée du garage Louis-Colin, plutôt qu’à l'entrée de la place de la Laurentienne à droite, montrent l'importance accordée aux accès indirects au campus.

Source: Université de Montréal - Paysagiste, [entre 1964 et 1976].

Photographie: Jacques Varry. Université du Québec à Montréal. Service des archives et de gestion des documents. Fonds d'archives Jean-Claude-La-Haye, 63P1/1004 (27.00)
Chacun des réseaux répond aux objectifs selon sa propre logique avec des défauts et des qualités. Le réseau souterrain, par exemple, se présentait comme une «composante majeure» qui «permet de concrétiser un des principaux objectifs de l'étude d'aménagement» (Archives UQAM-5). Malgré l'absence de liens clairs avec le réseau extérieur, certaines de ses qualités méritent d'être soulignées. L'harmonisation esthétique avec l'architecture, l'éclairage qui l'«humanise» ainsi que l'aménagement de percées visuelles et la présence du roc lui donnent une qualité d'expérience. Les conditions climatiques et la topographie difficile justifiaient l'implantation du réseau souterrain, en plus de s'inscrire dans la tendance des années 1960 d' «habiter» le souterrain. En effet, la construction de la Place Ville Marie, alors à peine achevée, donne le coup d'envoi à l'établissement d'un réseau souterrain qui, maintenant, caractérise Montréal. Le réseau souterrain du campus aujourd’hui, tout comme d'autres composantes dont on parlera plus loin, donne à voir des détails intéressants, dont la présence d'œuvres d'art, de plafonds en lattes de bois soigneusement agencées et de parois rocheuses reflétant la géomorphologie de la montagne.

À la lecture des rapports du plan directeur, on constate la richesse des idées sur les espaces libres, mais les plans montrent quant à eux un écart entre les intentions et la mise en espaces. On peut se demander si le nombre de plans pour exprimer la vision de «réseau devant relier les fonctions et s'intégrer à la ville» va à l'encontre de l'idée de «créer des liens organiques entre les diverses fonctions du campus, et d'en faire un tout vivant et intégré à la ville» (La Haye, 1964: 17). La notion du «tout» est justement difficile à cerner à l'aide des plans, tant les systèmes de circulation piétonne et véhiculaire sont dispersés sur plusieurs plans. Les circuits piétonniers apparaissent sur un plan, tandis que ceux des espaces verts et des véhicules automobiles sont sur un autre. De plus, les espaces libres dont il était question sont montrés sur le plan intitulé Le projet - les circulations piétonnes sous forme d'un remplissage sans nuance des espaces entre les bâtiments. En termes de représentation, on est loin d'y voir un parcours cohérent de places liées entre elles. On peut y sentir un paradoxe entre: séparer les fonctions (séparer les piétons des automobiles, le circuit intérieur du circuit extérieur) et créer un «tout organique», malgré un texte plutôt clair. 


\section{Le concours Vincent-Massey: illustration d'un campus convivial}

Le plan directeur du campus soumis au concours Vincent-Massey pour l'aménagement urbain de 1971 est sans doute l'illustration la plus représentative de l'idéalisme de La Haye et son équipe (figure 15). Dominés par une vision ludique des espaces libres, les documents du concours se distinguent de tous ceux dont nous venons de parler, à commencer par le graphisme, où abondent les personnages et la verdure. Tandis que les traits des plans de 1968 donnaient l'apparence de plans techniques, le graphisme du plan de concours détonne par l'exubérance graphique et par son aspect ludique. Inscrit dans un verdoyant ensemble, chaque espace extérieur est illustré et porte un nom propre: place des HEC, place du Droit, place des Sciences sociales, terrasse des Résidences, belvédère, etc. Que ce soit volontaire ou non, l'accent mis sur les espaces libres et sur les usagers coïncide avec les intentions de départ ainsi qu'avec les objectifs du concours. Le prix Vincent-Massey pour l'aménagement urbain visait à honorer les projets faisant preuve d'«excellence au niveau de l'environnement urbain» au bénéfice des citoyens (Gosselin-Geoffrion, 1975). Les prix soulignent les réalisations ayant eu un impact citoyen. Le graphisme répond ainsi aux objectifs, tout en reflétant les tendances graphiques de l'époque dans le domaine de l'aménagement où la présence de personnages et les traits de dessins superposés aux traits d'une écriture manuelle enrichissent la représentation. Notons que, selon toute vraisemblance, le prix Vincent-Massey pour l'aménagement urbain a été attribué uniquement à deux reprises, soit en 1971 et en $1975^{17}$. Le plan directeur du campus n’a pas été retenu, mais le dossier est notable puisqu'il montre une forme d'idéalisation du campus qu'entretenaient peut-être les concepteurs. On peut supposer que ce concours, en dehors des contraintes contractuelles, permettait aux concepteurs d'exposer un idéal du campus de l'Université de Montréal à une période où, socialement, on accordait une plus grande place à l'usager dans l'espace public.

\section{État actuel des lieux}

L'implication de La Haye et associés dans l'agrandissement du campus va au-delà de la planification puisque la firme réalisera plusieurs espaces libres, encore visibles de nos jours, et s'impliquera dans l'ensemble des travaux. Il en résulte une esthétique unifiée et urbaine dont certaines caractéristiques se retrouvent ailleurs sur le territoire de Montréal, comme l'usage du béton texturé. La Haye s'assurera de contrôler plusieurs aspects de la réalisation: «les aménagements d'ensemble [proposés] ne sauraient créer de problèmes insolubles aux architectes à qui [serait] confiée éventuellement la tâche de dresser des plans d'architecture» (Archives, UQAM-6). Le passage des recommandations à la réalisation sera assuré par un comité de suivi, le Comité consultatif d'architecture de l’Université de Montréal auquel Jean-Claude La Haye participera. Les aménagements extérieurs ont été sous la gouverne de sa firme sous forme de mandats indépendants, lui permettant de poursuivre sa vision ${ }^{18}$.

17 Parmi les réalisations primées en 1975, on compte les Jardins Prince-Arthur à Montréal, la promenade des Gouverneurs à Québec et le square Campbell à Scarborough.

18 La firme a en effet signé la majeure partie des aménagements de cette période. Les plans d'aménagement des espaces publics qui ont été consultés pour cet article se trouvent aux archives de la Direction des immeubles de l’Université de Montréal. 


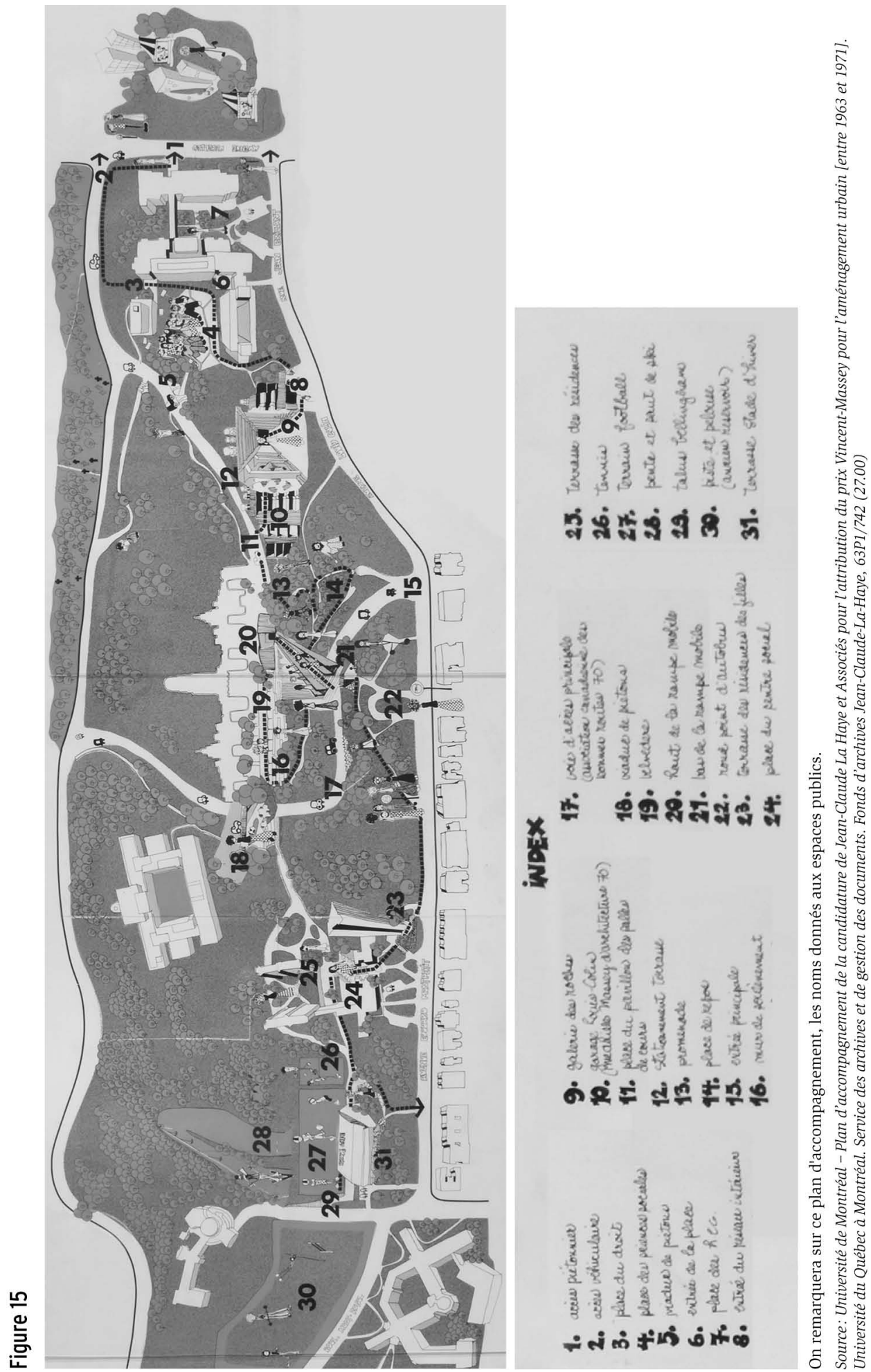


Parmi les nombreuses réalisations, soulignons le toit et les abords du garage LouisColin, le pourtour des pavillons des sciences humaines, la place de la Laurentienne, les abords du pavillon des sports et des résidences. Il accompagnera les firmes d'ingénieurs lors de la réalisation des voies de circulation, de l'éclairage et de diverses composantes puis prendra part au design du mobilier, de l'éclairage et de la signalisation. Cette large implication à toutes les étapes du projet a été possible grâce aux nombreux collaborateurs professionnels, dont Jean Ouellet, architecte, Douglas Harper et Serge Coitoux, architectes paysagistes, Lorrain, Tourigny, Dubuc \& Gérin-Lajoie ingénieursconseils et la firme E.R. Chagnon limitée ${ }^{19}$. Elle découle des convictions de Jean-Claude La Haye, pour qui la planification d'ensemble du campus précède et accompagne les réalisations en architecture et en architecture de paysage sur le campus.

\section{Figure 16}

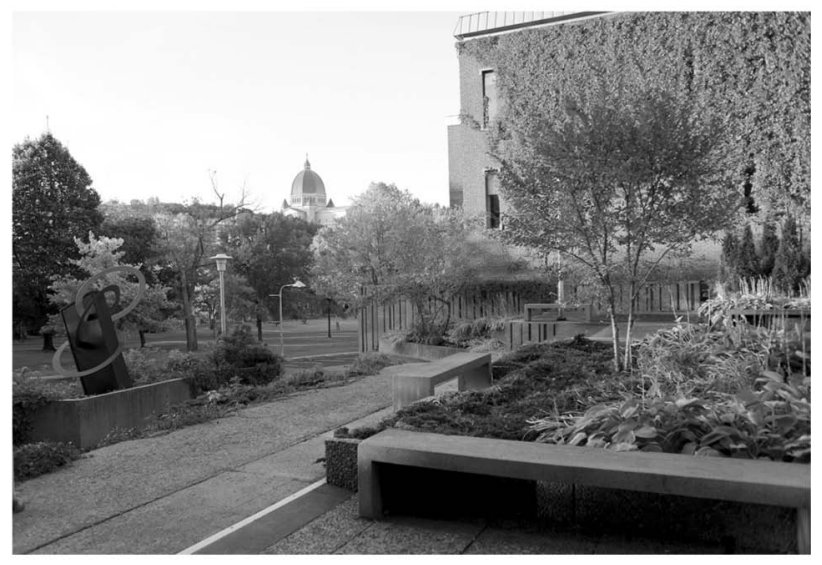

L’aménagement des abords sud du pavillon Maximilien-Caron a conservé son authenticité, notable par la présence des bancs en béton, des murets et des dalles de béton à agrégats exposés.

Photo: Denis Farley, 2010.

Figure 17

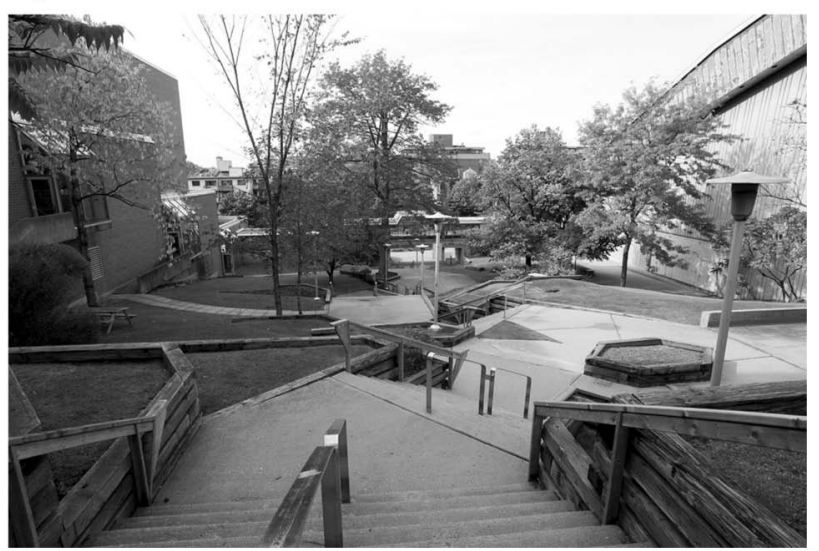

Le passage aménagé entre le Cepsum et le Stade d'hiver a gardé l'esprit initial de la réalisation de La Haye et associés.

Photo: Denis Farley, 2010.
Les apports de professionnels des différentes disciplines ont néanmoins favorisé une esthétique homogène. Les travaux d’ingénierie comme ceux de l'architecture de paysage tels les murets, les surfaces de béton et les murs de soutènement montrent des assemblages et des textures semblables: béton texturé, béton à agrégats exposés, béton brut, etc. À l'instar d'autres réalisations de l'époque à Montréal où les propriétés esthétiques du béton étaient mises en valeur, notamment pour l'architecture, l'expérimentation s'affichait dans les espaces extérieurs. Parmi les plus remarquables, on compte les abords sud du pavillon Maximilien-Caron et l'espace entre le stade et le Centre d'éducation physique et des sports de l'Université de Montréal (Cepsum), bien conservé et récemment rénové dans l'esprit de la réalisation initiale (figures 16 et 17). L'utilisation du bois et du béton texturé, l'emploi de formes angulaires et l'articulation de l'escalier à la

19 Douglas Harper fut l'un des fondateurs de l'École d'architecture de paysage de l'Université de Montréal. 
pente suivant des angles variés, tous ces aspects sont typiques de cette période. On peut en dire autant des murets de madriers de bois, identiques à ceux qu'on retrouve autour des pavillons Jean-Brillant et Lionel-Groulx. De plus, le motif répété de l'hexagone dessinant tantôt une fosse d'arbre, tantôt un motif de pavage apparaît à plusieurs endroits sur le campus, notamment sur le parvis du pavillon Thérèse-Casgrain et sur la place de la Laurentienne, comme ailleurs à Montréal. Ces exemples d'utilisation de matériaux et d'assemblage de formes sont représentatifs d'une pratique de l'architecture de paysage de cette période (figures 18 et 19).

\section{Figure 18}

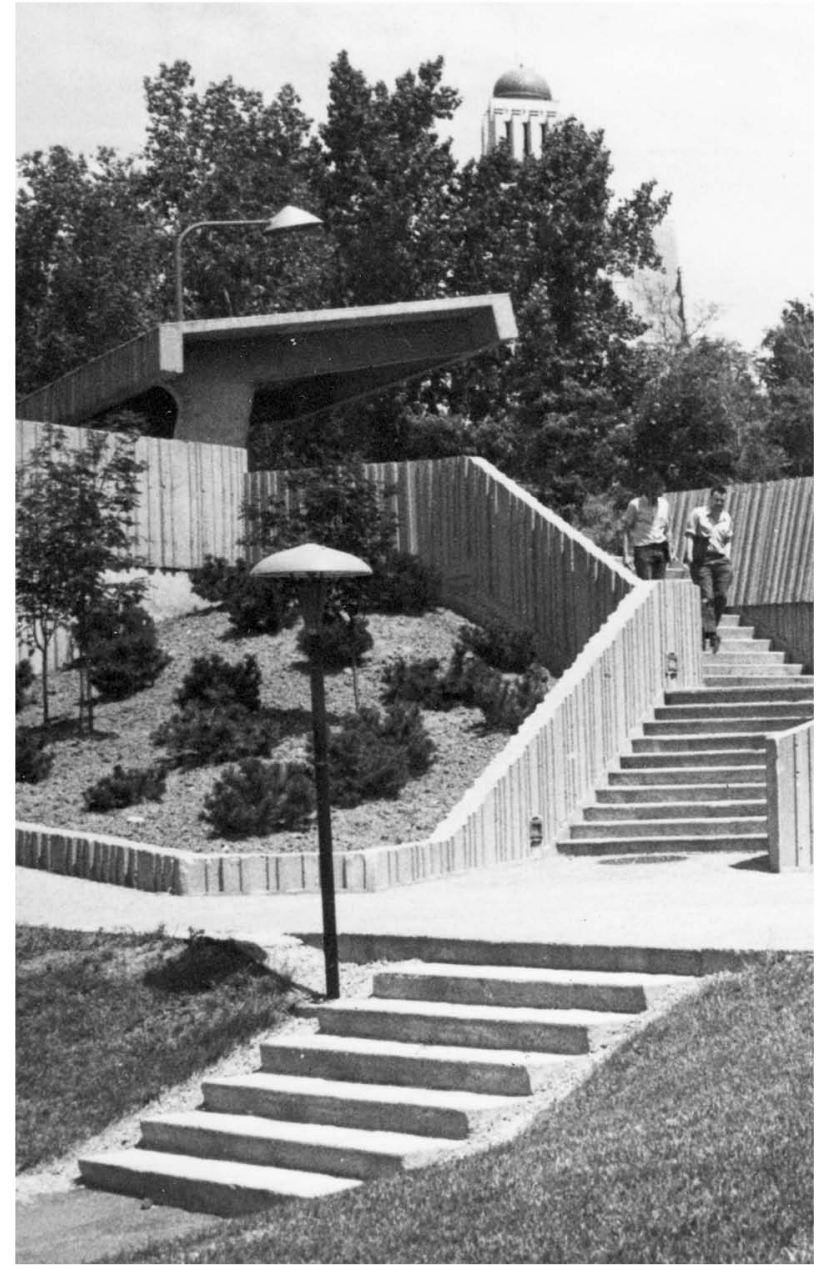

L'utilisation du béton texturé est notable dans les ouvrages réalisés par La Haye et associés dans les années 1960. Ce passage le long du pavillon Samuel-Bronfman en est un exemple.

Source: Université de Montréal - Plan d'ensemble [entre 1960 et 1980]. Université du Québec à Montréal. Service des archives et de gestion des documents. Fonds d'archives Jean-Claude-La-Haye, 63P1/734 (27.00)
Certains des aménagements ne sont pas convenablement conservés et d'autres ont été mal exécutés, mais plusieurs sont dignes d'attention. La place de la Laurentienne est probablement la plus méritante, si l'on en juge par sa valeur architecturale et historique (Cameron et al., 2008). Les bâtiments et la place, qui forment un ensemble homogène, témoignent de l'esthétique des espaces publics des années 1960 par leurs formes angulaires et l'utilisation du béton à agrégats exposés et texturés (figures 20 et 21). L'état de délabrement et la disparition d'une partie de la place lors de la construction de la bibliothèque des Lettres et sciences humaines poseront toutefois des défis importants lorsque le temps viendra de réaménager cet ensemble.

Parmi les autres détails intéressants, il faut retenir l'"équipement normalisé», dont une partie est illustrée en détail sur la planche intitulée Le projet

- les espaces verts. Les guérites, l'éclairage, les bacs à fleurs, les bancs, les paniers à déchets, les bornes de signalisation font partie de l'esthétique de cette période et 


\section{Figure 19}

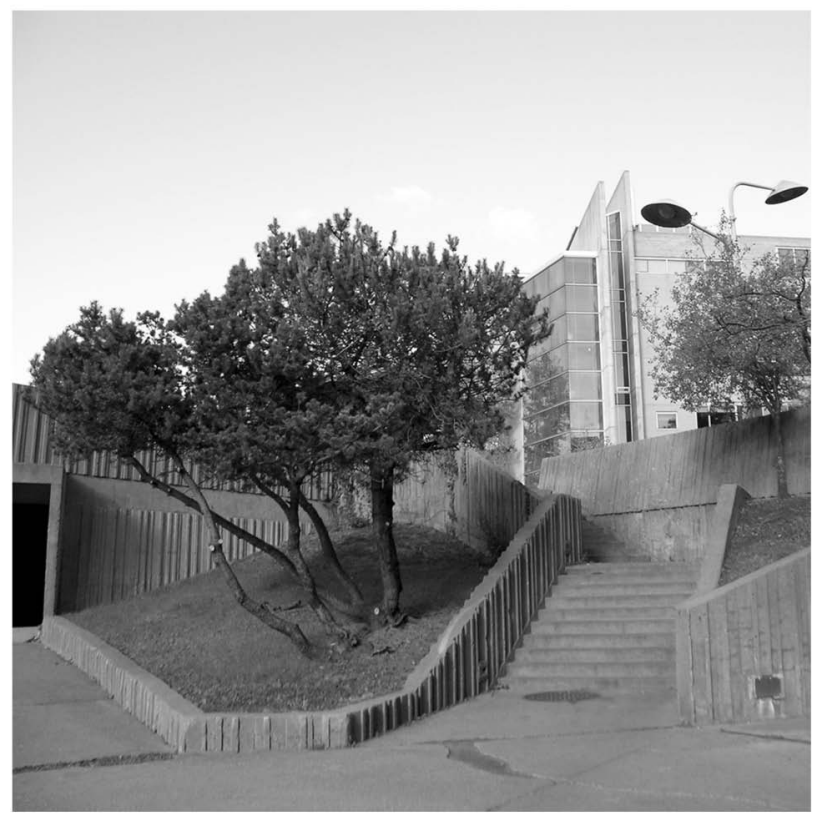

Le motif du pavage au pied du pavillon Thérèse-Casgrain ainsi que les bancs font partie de l'héritage architectural des espaces libres conçus par La Haye et associés au tournant des années 1970.

Photo: Nicole Valois, 2011.

\section{Figure 20}

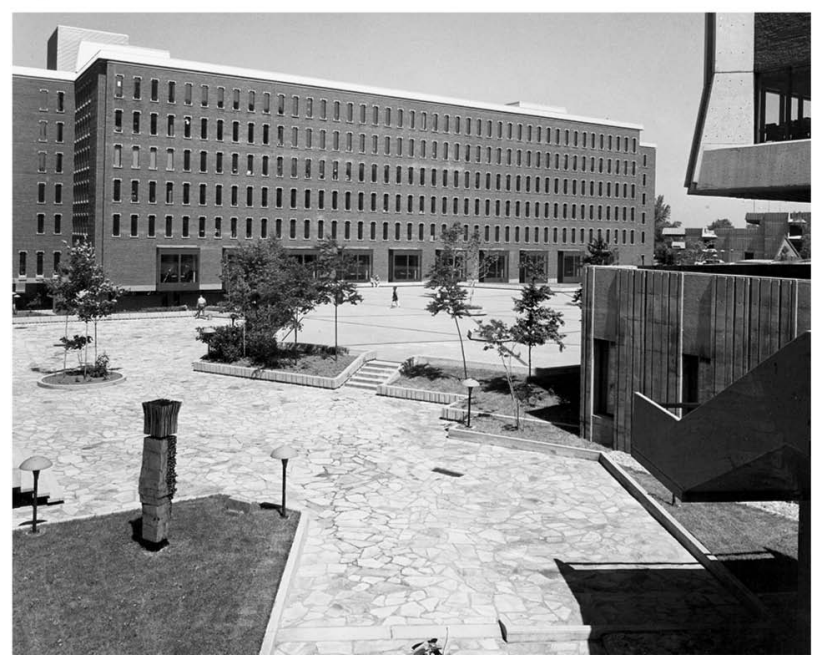

La place de la Laurentienne est l'une des réalisations les plus notables de cette période. Elle incarne très bien le principe de lieu de rassemblement pour la communauté universitaire, fortement lié à la composition architecturale des édifices qui l'entourent.

Source: Université de Montréal - Paysagiste [entre 1964 et 1971].

Photographie: Jacques Varry. Université du Québec à Montréal. Service des archives et de gestion des documents. Fonds d'archives Jean-Claude-La-Haye, 63P1/1008 (27.40) de la vision d'ensemble de l'équipe de La Haye. Cette attention accordée au mobilier, combinée aux indications sur les espaces à aménager, reflète encore une fois l'approche pluridisciplinaire chère à JeanClaude La Haye.

Malheureusement, d'autres aménagements paysagers de cette période sont en très mauvais état. Les problèmes spatiaux générés par les constructions subséquentes et la détérioration des matériaux due au manque d'entretien, alors que ces ouvrages sont encastrés dans de fortes pentes sujettes à l'érosion, sont à ce point critiques qu'à certains endroits la démolition semble inévitable. Le moment est venu d'évaluer ce qui mérite d'être conservé versus ce qui mérite d'être restauré, remplacé ou corrigé en regard de l'état des lieux, mais aussi en regard du témoignage d'un savoirfaire en planification et en aménagement paysager qui prenait naissance au Québec, dans les années 1960. 


\section{Figure 21}

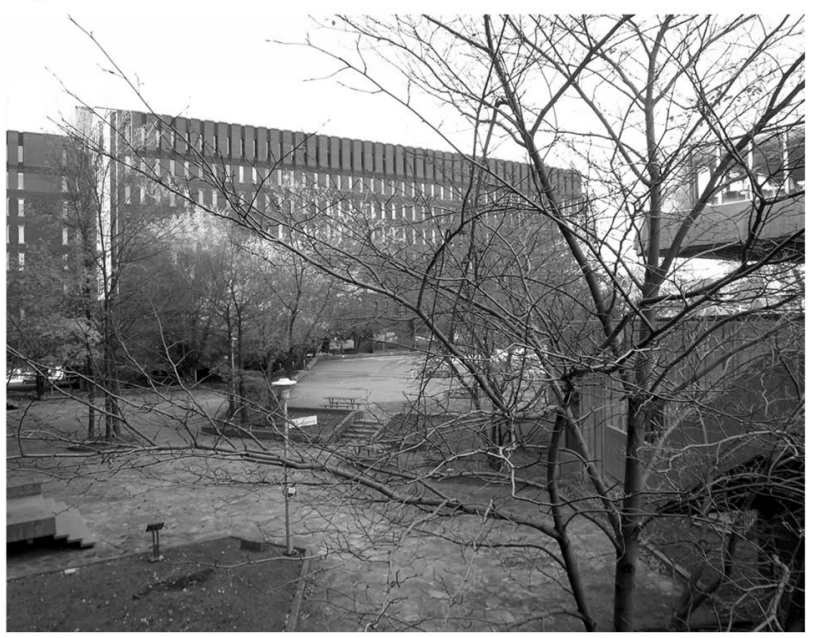

Vue actuelle de la place de la Laurentienne dont l'apparence reste inchangée. Photo: Nicole Valois, 2011.

\section{Conclusion}

Cette période où le campus se tourne vers la modernité et où les premiers aménagements paysagers prennent véritablement forme est importante sur les plans de l'histoire du campus et de son secteur ainsi que des professions d'aménagement. On retiendra que l'Université de Montréal fut l'une des premières institutions à s'implanter sur le flanc nord de la montagne de manière aussi marquée. La tour de l'édifice principal, tel un repère dans la ville, marque ce territoire. La présence du campus a agi sur le développement tout autant que son évolution a répondu à l'élan de modernité qui animait Montréal. Les aménagements extérieurs des années 1960 incluant les voies véhiculaires, les sentiers et les places incarnent cet élan par leurs attributs esthétiques et l'esprit de réseau d'espaces libres vu comme un moyen d'unifier les espaces physiques et les gens qui les utilisent.

La planification à long terme du campus par la firme de La Haye relève d'une pratique émergente en termes de techniques de planification qui a donné naissance à de nombreux campus en Amérique. L'ampleur du mouvement a fait en sorte de positionner les campus parmi les typologies de l'architecture de paysage, dans le corpus de l'histoire de l'architecture moderne en Amérique du Nord (Treib, 2003). À ce titre appartiennent sans doute celui de l'Université de Montréal de même que d'autres campus canadiens comme ceux de l’Université Laval, l’Université de Waterloo ou l'Université de Guelph.

Les premiers aménagements paysagers planifiés du campus de l'Université de Montréal apparaissent au moment où l'architecture de paysage et l'urbanisme prennent leur l'envol au Québec et que s'instaure l'enseignement de leur pratique à la même université, au sein de la Faculté de l'aménagement. L’apport de plusieurs disciplines que requiert le processus de planification allant jusqu'aux aménagements spécifiques, comme en témoigne le cas du campus, est propre à l'esprit de regroupement des disciplines à la Faculté de l'aménagement. En tant qu'individu, La Haye était au cœur de 
ces mouvements fondateurs, en enseignement et dans la pratique. Le plan directeur du campus dont il a dirigé la réalisation a été un outil de planification permettant de tisser les liens entre les composantes physiques du lieu pour créer un grand espace singulier, fonctionnel, jusque dans les moindres détails des espaces extérieurs. Que cette portion importante du campus ait été réalisée dans une période aussi foisonnante par un concepteur pionnier de l'urbanisme aussi engagé dans la reconnaissance de la profession et dans le développement du Québec mérite d'être souligné.

L'envers de la médaille montre toutefois l'héritage d'un campus fortement marqué par l'importance de la voiture, propre aux tendances urbanistiques de cette période. Certains des aménagements contreviennent à la vision de grande liberté de déplacements prônée par les plans directeurs, tant la difficulté de se déplacer à pied en toute sécurité est grande. Les transformations continuelles et le manque d'entretien des espaces sont aussi des problèmes réels auxquels l'Université doit s'attaquer en ayant à l'esprit la mise en valeur de ce patrimoine et le maintien de principes liés au caractère du site.

Les campus sont souvent comparés à un microcosme de la ville, évoluant au gré des changements de société et où le passage du temps renvoie aux tendances stylistiques et fonctionnelles, mais aussi aux impératifs d'entretien et de moyens financiers. Malgré le vieillissement des aménagements extérieurs de cette période faste du campus (sans parler des édifices) et dans un contexte de sous-financement, procéder à leur réaménagement peut sembler peu prioritaire, d'autant plus qu'un nouveau campus accueillera prochainement l'expansion du campus actuel de l'Université de Montréal. Néanmoins, celle-ci entreprendra de grands travaux de réaménagement du pourtour du pavillon principal dans les années à venir ${ }^{20}$. La préservation des éléments significatifs de cette période, dont la vision planificatrice et systémique s'inscrit pour la toute première fois dans son histoire, pourrait faire partie de ce processus de renouveau, et ce, considérant qu’à Montréal les exemples sont peu nombreux et documentés. Les projets auxquels collabore la Ville de Montréal, tels le parc du Troisième sommet et le Chemin de ceinture, ne semblent pas inclure pour l'instant cette considération. Pourtant, il y a lieu de croire que l'accessibilité accrue du campus par le public permettra de le relier davantage à la ville. En somme, les projets en cours et futurs offrent la chance de créer un véritable campus et d'actualiser son image fondée, sous l'impulsion de La Haye et associés, sur un réseau d'espaces libres dans le contexte naturel et hors du commun qu'est la montagne.

20 Ce projet est, à ce jour, vraisemblablement à l'étape préliminaire, l’Université ayant lancé un appel d'offre auprès des professionnels au printemps 2012 pour évaluer la faisabilité des travaux. 


\section{Bibliographie}

ARCHIVES UQAM - 1. Service des archives et de gestion des documents, fonds d'archives Jean-Claude-La-Haye, 63P1/728. L'Université aujourd'hui et demain. Dépliant produit par l'Université de Montréal dans le cadre de la campagne du cinquantenaire. Montréal, avril 1967.

ARCHIVES UQAM - 2. Service des archives et de gestion des documents, fonds d'archives Jean-Claude-La-Haye, 63P1/719. Lettre de Jean-Claude La Haye adressée à Monseigneur Irénée Lussier. Montréal, datée du 4 janvier 1960.

ARCHIVES UQAM - 3. Service des archives et de gestion des documents, fonds d'archives Jean-Claude-La-Haye, 63P1/719. Trudel, Jacques. Service d'urbanisme, AMUR, Université de Montréal, une nouvelle analyse. Montréal, février 1967.

ARCHIVES UQAM - 4. Service des archives et de gestion des documents, fonds d'archives Jean-Claude-La-Haye, 63P1/719, Dimakopulos, Dimetri et al. Formulation des conditions préalables à une politique d'ensemble de développement. Montréal, 1966.

ARCHIVES UQAM - 5. Service des archives et de gestion des documents, fonds d'archives Jean-Claude-La-Haye, 63P1/719. Réseau piétonnier intérieur. Document relié; date et auteur inconnus. Montréal.

ARCHIVES UQAM - 6. Service des archives et de gestion des documents, fonds d'archives Jean-Claude-La-Haye, 63P1/719. Lettre de Jean-Claude La Haye adressée à André Casaubon de l'Université de Montréal. Montréal, 12 janvier 1963.

BIZIER, Hélène-Andrée (1993) L'Université de Montréal: la quête du savoir. Montréal, Libre expression.

BOISVERT, André (2003) Conversations avec les pionniers et pionnières de l'urbanisme au Québec. Urbanité, vol. 2, n³, p. 7-12.
CAMERON, Christina, DÉOM, Claudine et VALOIS, Nicole (2008) L'étude des valeurs patrimoniales du campus principal de l'Université de Montréal. Chaire de recherche du Canada en patrimoine bâti, Université de Montréal.

CAMERON, Christina, DÉOM, Claudine et VALOIS, Nicole (2010) Le patrimoine architectural et paysager de l'Université de Montréal. Montréal, Presses de l'Université de Montréal.

CORMIER, Lise (1990) Des parcs aux espaces verts. Continuité, no 1 , p. 52-55.

DOBER, Richard P. (1963) Campus planning. New York, Reinhold Publishing Corporation.

FIFE, Ed, CRAWFORD, Pleasance et ELIAS, Ina (2012) Landscape architecture. The Canadian Encyclopedia. Historica Foundation. [En ligne]. http://www.thecanadianencyclopedia.com/articles/fr/ architecture-paysagere.

GARCIA, Karine et GARCIA, Sylvain (2006) Campus de l'Université de Montréal et secteur adjacent - Étude typo-morphologique. Montréal, Bureau du mont Royal de la Ville de Montréal.

GOSSELIN-GEOFFRION, Hélène (1975) Prix Vincent-Massey pour l'aménagement urbain. Architecture Concept, vol. 30, nº330.

GOURNAY, Isabelle (1990) Ernest Cormier et l'Université de Montréal. Montréal, Centre canadien d'architecture.

LANDRY, Jean (1992) Le système des espaces libres de la ville de Montréal. Dans Remiggi, Frank W. et Gilles Sénécal (dir.) Montréal. Tableaux d'un espace en transition. Montréal, Association canadiennefrançaise pour l'avancement des sciences, Les Cahiers scientifiques, $\mathrm{n}^{0} 76$.

LA HAYE, Jean-Claude (1964) Rapport d'accompagnement du plan général d'aménagement de l'Université de Montréal. Montréal, Université de Montréal. 
LA HAYE, Jean-Claude (1968a) Université de Montréal: le deuxième plan directeur d'aménagement. Montréal, Université de Montréal.

LA HAYE, Jean-Claude (1968b) Rapport de la Commission provinciale d'urbanisme. Québec, Gouvernement du Québec.

MARSAN, Jean-Claude (1974) Montréal en évolution: historique du développement de l'architecture et de l'environnement montréalais. Montréal, Fides.

MARSAN, Jean-Claude (2009) L'avenir du campus de l'Université de Montréal. Forum, vol. 13, n² 2, p. 4-7.

OUELLET, Jean, BOISVERT, Jean-Claude et PARÉ, Jean (1995) Plan directeur: campus de l'Université de Montréal. Montréal, Université de Montréal.

PAINE, Cecelia (dir.) (1998) Cinquante ans d'architecture de paysage: l'Association des architectes paysagistes du Canada: compte rendu officiel du 50 Congrès tenu en 1984. Guelph, Université de Guelph.

QUÉBEC, Ministère de l'Éducation (19651966) Rapport de la Commission royale d'enquête sur l'enseignement dans la province de Québec. Québec, Les publications du Québec.

TELLIER-COHEN, Loraine (2003) Rencontre avec Jean-Claude La Haye. Urbanité, vol. 2, no3, p. 40-43.

TREIB, Marc (2003) Open spaces and landscapes: Some thoughts on their definition and preservation. Dans Ron van Oers (dir.) World heritage paper 5 - Identification and Documentation of Modern Heritage. Paris, UNESCO World Heritage Center, vol. 5, p. 135-137.

TRUDEL, Jacques (2011) Le développement de l'Université de Montréal: un rendezvous manqué avec l'urbanisme? Urbanité, Hiver, p. 49-50.

TURNER, Paul Venable (1984) Campus: An american planning tradition. Cambridge, Architectural history foundation/MIT Press.
VILLE DE MONTRÉAL (1944) Plan directeur: urbanisation de Montréal. Montréal, Service d'urbanisme.

VILLE DE MONTRÉAL (1955) Espaces libres: plan directeur de Montréal. Montréal, Service d'urbanisme, Division du plan directeur. 